\title{
Protocol
}

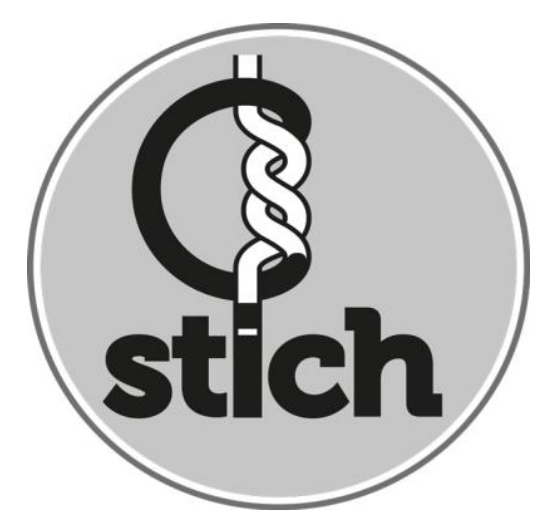

\section{Cerclage Suture Type for an Insufficient Cervix and its effect on Health outcomes (C-STICH)}

UNIVERSITYOF BIRMINGHAM

\section{NTS}

Birmingham Women's and Children's NHS Foundation Trust 


\section{C-STICH Trial Management Group}

\section{Chief Investigator:}

\section{Mr. Philip Toozs-Hobson}

Consultant Urogynaecologist

Birmingham Women's and Children's NHS

Foundation Trust, Birmingham Women's Hospital, Mindelsohn Way, Edgbaston

Birmingham B15 2TG

Email: philip.toozs-hobson@nhs.uk

\section{Trial Coordination and Statistics:}

Dr Jane Daniels

Contact through trials office at

cstich@trials.bham.ac.uk

Tel: 07958541660

\section{Mr Lee Middleton}

Birmingham Clinical Trials Unit Institute of Applied Health Research University of Birmingham B15 2TT

Email: l.j.middleton@bham.ac.uk

Tel: 01214159117

\section{Mr Konstantinos Tryposkiadis} Birmingham Clinical Trials Unit Institute of Applied Health Research University of Birmingham B15 2TT Email: k.tryposkiadis@bham.ac.uk

\section{Ms Joy Rahman}

Birmingham Clinical Trials Unit Institute of Applied Health Research University of Birmingham B15 2TT Email: j.k.rahman@bham.ac.uk Tel: 01214147023

\section{Clinical Research Fellow:}

\section{Dr Victoria Hodgetts}

Email: v.a.h.morton@bham.ac.uk

Birmingham Women's Hospital,

Mindelsohn Way, Edgbaston

Birmingham B15 2TG

Tel: 01216272779

\section{Parent Representation and Support:}

Janet Scott - PPI Representative, Research and Prevention Manager, Sands.

Tel: 02074365881

Email: janet.scott@uk-sands.org

Laura Flemming - Senior Midwife, Bereavement Services, Birmingham Women's Hospital Tel: 07733338184

Email: Laura.Flemming@bwnft.nhs.uk

\author{
Microbiology Lead Investigator: \\ Dr Jim Gray \\ Birmingham Women's Hospital \\ Birmingham B4 6NH \\ Email: Jim.Gray@bch.nhs.uk \\ Tel: 01216272727
}

\section{Clinical Lead Investigators}

\section{Mr. Philip Toozs-Hobson \\ Consultant Urogynaecologist \\ Contact as above}

Dr Andrew Ewer - Clinical Academic in Neonatal Medicine, Queen Elizabeth Hospital, Birmingham Email: a.k.ewer@bham.ac.uk

Tel: 01214721377

Mr. Christoph Lees - Lead Consultant in Maternal Foetal Medicine, Imperial College, London Email: christoph.lees@imperial.nhs.uk

Tel: 02083833998

Prof Jane Norman - Chair, Obstetrics and Gynaecology, University of Edinburgh Email: jane.norman@ed.ac.uk Tel: 01312422694

Mr Mark Slack - Consultant Urogynaecologist, Addenbrooke's Hospital, Cambridge Email: mark.slack@addenbrookes.nhs.uk Tel: 01223586740

Prof Andrew Shennan - Clinical academic specialising in pre-term birth and cerclage, King's College, London

Email: andrew.shennan@kcl.ac.uk Tel: 02071883639

Mr Nigel Simpson - Consultant Gynaecologist Leeds General Infirmary

Email: n.a.b.simpson@leeds.ac.uk

Tel: 01133922729

\section{Professor Khaled Ismail}

Birmingham Women's Hospital

Contact via Trial Co-ordinator

Dr Fidan Israfil-Bayli

Good Hope Hospital

Email: f.israfilbayli@nhs.uk 


\section{Trial Steering Committee}

Independent Members:

- Prof Harry Gee (Chair) University of Warwick Harry.gee1@gmail.com

- Prof Christine Kettle Professor Emerita, Women's Health, Staffordshire University, Stoke-on-Trent, ST4 2DE chrisk16@tiscali.co.uk

- Dr Jon Dorling

Clinical Associate Professor, Faculty of Medicine \& Health Sciences, Queen's Medical Centre, Nottingham, NG7 2UH

Jon.Dorling@nottingham.ac.uk

- Ms Sarah Louise Machin, Independent PPI Representative, Contact via Trial Co-ordinator

Non-independent Members:

- Mr Philip Toozs-Hobson (Chief Investigator)

- Professor Jane Daniels

\section{Data Monitoring and Ethics Committee}

For interim analysis and response to specific concerns:

- Professor Marian Knight (Chair)

National Perinatal Epidemiology Unit (NPEU), Oxford, OX3 7LF marian.knight@npeu.ox.ac.uk

- Professor Douglas Tincello (Obstetrics \& Gynaecology - Leicester) Leicester General Hospital, Gwendolen Road, Leicester LE5 4PW dgt4@le.ac.uk

- Mr Graeme MacLennan (Senior Statistician - University of Aberdeen) Health Sciences Building, University of Aberdeen, AB25 2ZD g.maclennan@abdn.ac.uk 


\section{C-STICH Trials Office}

Birmingham Clinical Trials Unit, Institute of Applied Health Research, Public Health Building, the University of Birmingham, Birmingham B15 2TT Telephone: 01216872310 (Voicemail outside office hours)

Fax: $01214159136 \quad$ E-mail: bctu@bham.ac.uk

Co-ordination: Ms Joy Rahman: J.K.Rahman@bham.ac.uk

Data Management: Mr Peter Price: p.j.price@bham.ac.uk

CStich@trials.bham.ac.uk; Telephone 01214147023

www.birmingham.ac.uk/C-STICH

Statistics: Mr Lee Middleton, Trainee: Mr Konstantinos Tryposkiadis

Clinical queries should be directed during office hours to an appropriate member of the Management Group. Other queries should be directed to the C-STICH Trials Office.

FOR RANDOMISATIONS

Online at https//www.trials.bham.ac.uk/CSTICH (24 hours)

TELEPHONE: 08009530274 (UK)

$0900-1700$ hrs Monday to Friday

Alternatively, by fax on: 01214159136 (UK) 


\section{Previous protocol versions and summary of changes:}

\section{Version 1.0 to 1.1:}

Correction of minor typographical errors and clarification of inclusion and exclusion criteria.

\section{Version 1.1 to 2.1:}

To change the study Sponsor from University of Birmingham to Birmingham Women's NHS FT.

To remove University of Birmingham logos and insert Birmingham Women's NHS FT logos.

To change the study Chief Investigator from Professor Khaled Ismail to Mr Philip Toozs-Hobson.

To change the title of the study from 'Cerclage Suture Type for an Insufficient Cervix and its effect on Health Outcomes Trial: a randomised controlled Trial of monofilament versus braided sutures for insufficient cervix' to 'Cerclage Suture Type for an Insufficient Cervix and its effect on Health Outcomes (C-STICH)'.

To clarify the inclusion and exclusion criteria.

To remove the necessity for reporting non-serious adverse events.

To add hospitals in Ireland as settings.

To correct minor typographical and grammatical errors.

\section{Version 2.1 to 3.0:}

To allow consent of patients to C-STICH by all suitably qualified and designated clinical staff.

To clarify the criteria for co-enrolment.

To add a further criterion for reporting serious adverse events.

To replace MREC with REC as multi-research ethics committees no longer exist.

\section{Version 3.0 to 4.0:}

To clarify the secondary maternal and neonatal outcomes.

To alter the end of trial definition.

To change the microbiology processing information.

To clarify adverse events and serious adverse events.

To detail arrangements for the trial monitoring and clarification of responsibilities for this.

To amend blinding conditions.

To clarify participation in other trials including CTIMP trials.

To remove the discussion of cerclages being performed between 12-22 weeks in the pregnancy as it has caused confusion over eligibility criteria and there are no gestational limits for eligibility with the trial.

To change the requirement to send case report forms to the trial office if entering online. 


\section{Version 4.0 to 5.0:}

To change the name of Sponsor from Birmingham Women's NHS Foundation Trust to Birmingham Women's and Children's NHS Foundation Trust as the two Trusts have now merged.

To further clarify the secondary outcomes to be analysed.

To add birth weight and small for gestational age as secondary outcomes to be analysed.

The following has been broken down so that it is clearer:

- Adverse events: suture related cervical tears, chorioamnionitis, maternal pyrexia of $38^{\circ} \mathrm{C}$, systemic infection requiring antibiotics (infection parameters based on Centre for Disease Control / National Healthcare Safety Network [CDC / NHSN] guidance).

The minimisation procedure for randomisation has been clearly defined.

To remove the necessity to take a high vaginal swab within 5 days as this is no longer considered important.

'C-STICH staff member' has replaced 'nurse/midwife/clinician' where applicable to prevent ambiguity over which staff members can perform specific roles.

Both 'obstetric and gynaecology' units have been used as participants are recruited at either.

To add to the ethical and Trust Management Approval section 'Confirmation of Capacity and Capability' to allow for the recent changes to HRA for sites in England.

To change Health and Social Care Information Centre to NHS digital as the name of this national provider of health information for England and Wales has now changed. To add ISD Scotland as a source where we obtain death information for the babies in Scotland.

To add that, where it is unknown as to which hospital the mother has delivered her baby, the mother's GP will be contacted and asked to provide details of the delivery hospital.

To add continuing care sites as a source, other than the recruiting site, where we will collect data if mothers deliver their baby at a site outside the recruiting centre and where the delivery hospital is not participating in C-STICH.

To add that where data is unobtainable from either the recruiting site or the continuing care site, it will be sought from the NHS Digital (England and Wales) or ISD (Scotland).

The patient pathway flowchart has been replaced by one without a version number on as it did not match the protocol version.

Details have been changed for one DMEC member. 
Summary and Declarations:

Protocol Version $\quad 5.0(23 r d$ March 2017)

Previous Versions V1.0, V1.1, V2.1, V3.0 V4.0

ISRCTN 15373349

Funding Body: NIHR (HTA) Programme grant Number: 13/04/107

Sponsor: Birmingham Women's and Children's NHS Foundation Trust (BWCNFT)

\section{Chief Investigator: Mr Philip Toozs-Hobson}

The BWCNFT is responsible for obtaining the necessary approvals and governance. The Trial Management Group is jointly responsible for overseeing good clinical practice (GCP) and the Principal Investigators are responsible for obtaining informed consent and care of the participants.

\section{Chief Investigator's Declaration:}

The investigators and the Trial Sponsor have discussed this protocol. The investigators agree to perform the investigation and to abide by this protocol except in case of medical emergency or where departures from it are mutually agreed in writing.

Signature:

Date:

$\underline{\mathrm{DD} / \mathrm{MM} / \mathrm{YYYY}}$

Mr Philip Toozs-Hobson

Chief Investigator on behalf of the Trial Management Group. 
Principal Investigator's Declaration:

Site:

Principal Investigator:

Title:

Cerclage Suture Type for an Insufficient Cervix and its effect on Health Outcomes (C-STICH)

Version: $\quad 5.0$

I confirm I have received, read and understood the aforementioned version of the trial protocol. I confirm my team and I will adhere to this version of the protocol following receipt of the required local approvals.

Signature:

Date:

The Principal Investigator should sign this page and return a copy of this page to the C-STICH Trial Office 
Abbreviations

$A E$

AR

ASR

BCTU

$\mathrm{Cl}$

DMEC

GCP

GMP

GP

ISRCTN

REC

ONS

PI

PIS

RR

SAE

SOP

TMG

TSC
Adverse event

Adverse reaction

Annual Safety Report

Birmingham Clinical Trials Unit at the University of Birmingham

Chief Investigator

Data Monitoring and Ethics Committee

Good Clinical Practice

Good Manufacturing Practice

General Practitioner

International Standard Randomised Controlled Trial Number

Research Ethics Committee

Office for National Statistics

Principal Investigator - the local lead investigator for the C-STICH Trial

Participant Information Sheet

Relative Risk

Serious Adverse Event

Standard Operating Procedure

Trial Management Group

Trial Steering Committee 


\section{Trial summary}

Title

Cerclage Suture Type for an Insufficient Cervix and its effect on Health outcomes (C-STICH)

\section{Settings}

Obstetric and gynaecology departments in hospitals within the UK.

\section{Trial Design}

A multi-centre, open, randomised controlled trial of 900 women presenting at obstetric units and deemed to be at risk of an insufficient cervix, and scheduled to be treated by cervical cerclage.

\section{Aims}

1. To examine the effect of using a monofilament suture material compared with a braided suture material on pregnancy loss rate (defined as miscarriage, stillbirth, neonatal death in the first week of life) and neonatal mortality up to one month post-delivery in women deemed to be at risk of an insufficient cervix and treated with cervical cerclage.

2.

- To assess the effect of suture material on other pregnancy and neonatal outcomes

- To explore the variation in effect between McDonald's and Shirodkar's cerclage, especially with reference to bladder dissection

- To explore the variation in effect between the indication for cerclage

- To produce advice and a video clip to illustrate best practice in cerclage stitch insertion and removal

\section{Target Population}

Women over 18 years old with a singleton pregnancy and presenting with indications for cervical cerclage.

\section{Health Technologies Assessed}

Monofilament or braided suture material. 


\section{Lay Summary}

Every year approximately 3750 women in the UK will have complications where their cervix (the neck of the womb) becomes loose and opens during the early months of pregnancy. This can require a stitch being sewn into the cervix in an attempt to keep it closed. This is often referred to as 'cervical suture' or 'cervical cerclage'. If this procedure is not performed the cervix can open too early and can result in a miscarriage or premature birth. Inserting a stitch into the cervix does not guarantee to keep the cervix closed, but it can sometimes allow the pregnancy to continue for a few more weeks.

The stitches used for this procedure are available in different sizes and materials. Some of the stitch threads are made from a single, smooth fibre (e.g. nylon) while others are composed of many fibres which are woven to form a fine braided or net-like structure. A survey of consultants in the UK has shown most use braided threads when they stitch the cervix merely because it is the traditional material used and because it is thought to offer strength and enhanced support to an otherwise loose cervix. However, this survey also revealed that some surgeons thought that bacteria could grow more easily in the spaces of the braided thread than on the surface of the monofilament line. This could increase the risk of infection which might cause an early labour. It is therefore essential to investigate whether thread-type used for stitching the cervix increases or decreases risk of infection.

The C-STICH study will therefore compare outcomes from the use of either smooth or braided stitches during this procedure. The results of this study can potentially save the lives of more than 300 babies a year in the UK alone who would otherwise be at risk of severe prematurity or miscarriage.

The best way to compare the two methods of treatment is to undertake a clinical trial where the nature of the stitch used is decided randomly. Computer software, specifically designed for this purpose will be used at a specialised unit at the University of Birmingham (Birmingham Clinical Trials Unit).

Eligible pregnant women can opt to be part of the C-STICH study if they are due a planned stitch in their cervix during their pregnancy. Apart from the type of thread used, participants in the C-STICH study will receive identical medical treatment to those not taking part in the study. Several pregnancy outcomes will be collected though the central outcome for the study, as decided by the groups of women consulted, will be the risk of losing a baby during pregnancy or within a week of birth. Information will also be collected concerning the number of weeks pregnancy lasted prior to birth; whether the baby was admitted to a Neonatal Unit; the length of stay in the unit and any sign of vaginal or womb infection. 


\section{CONTENTS}

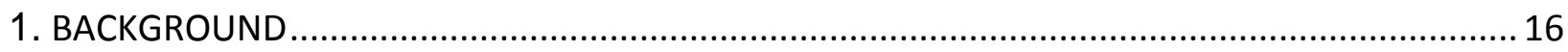

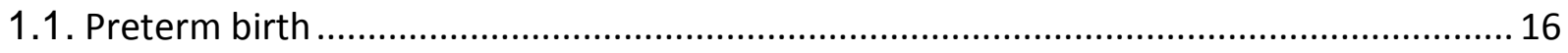

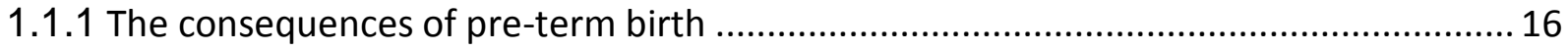

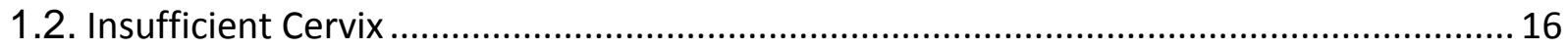

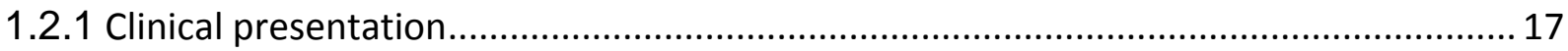

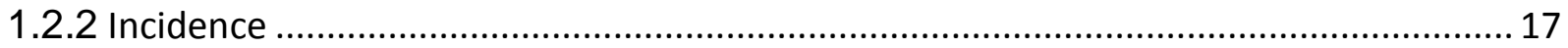

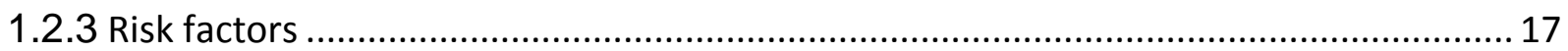

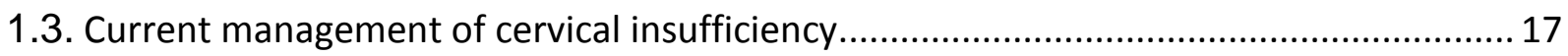

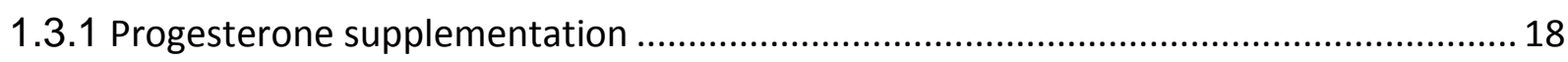

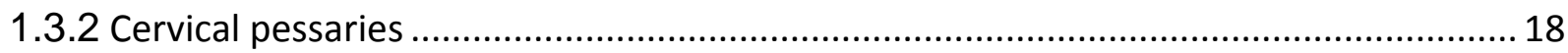

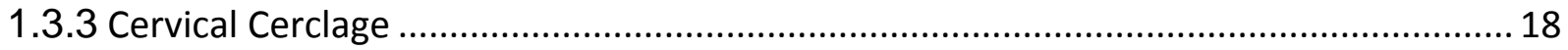

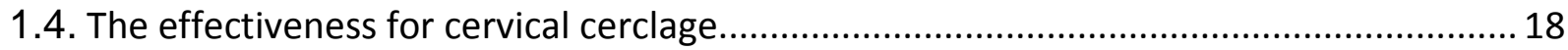

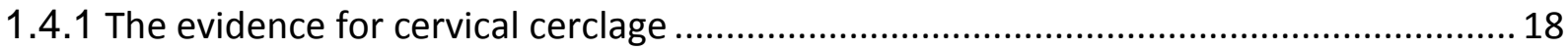

1.4.2 The influence of the suture material in gynaecological surgery.................................. 20

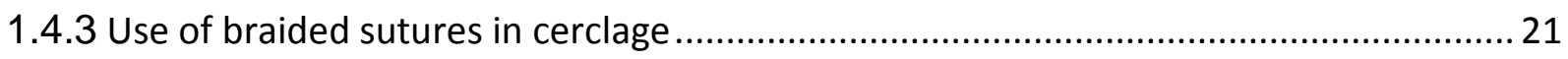

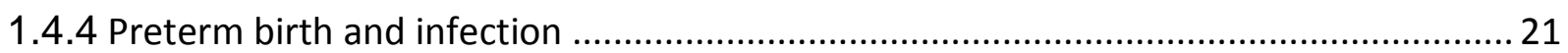

1.5. The evidence for effectiveness of the suture material used for cervical cerclage ........... 21

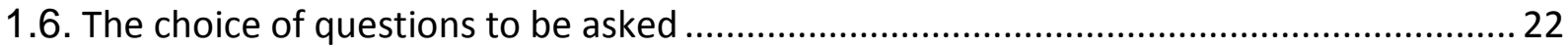

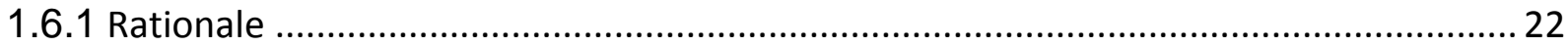

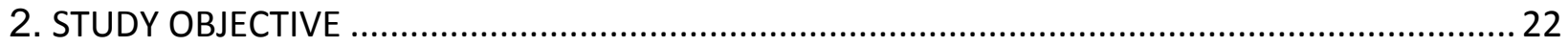

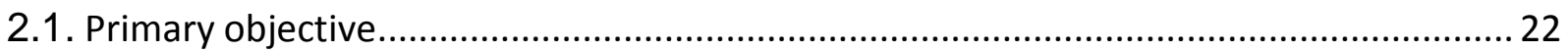

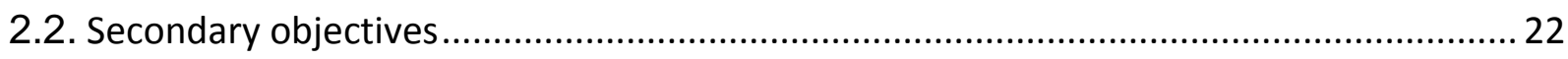

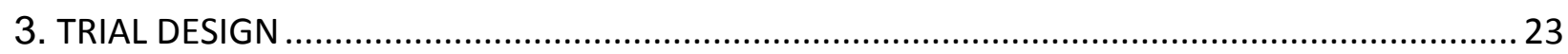

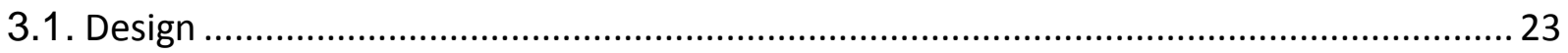

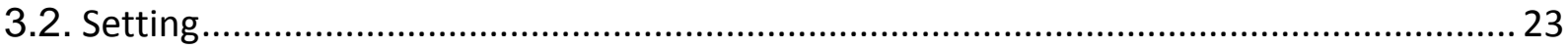

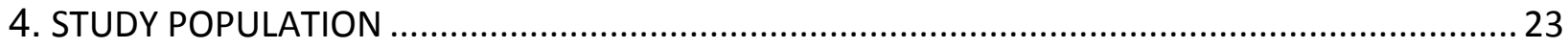

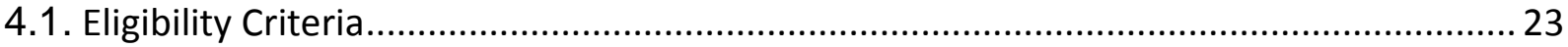

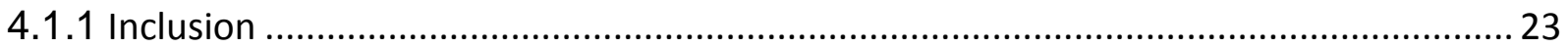

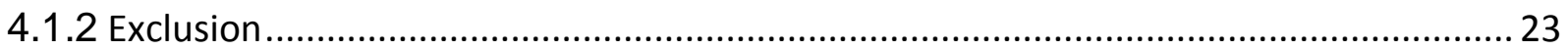

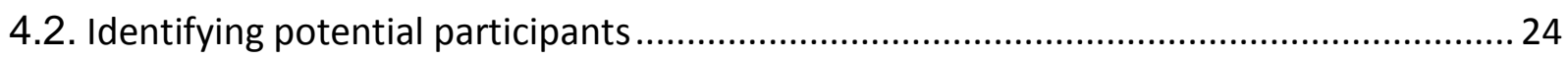

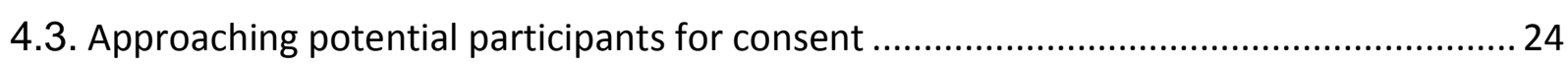

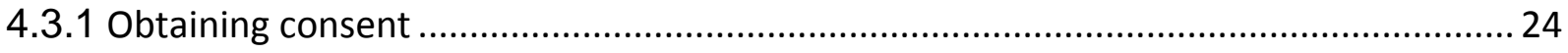

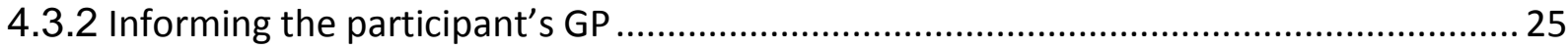




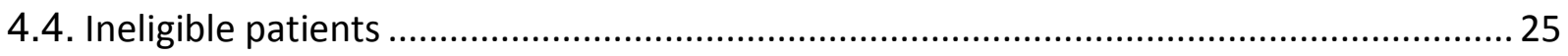

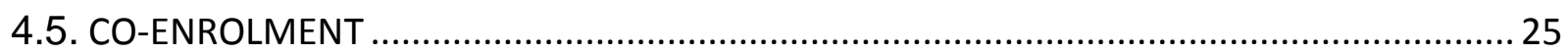

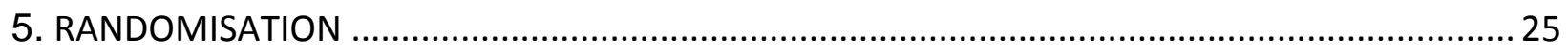

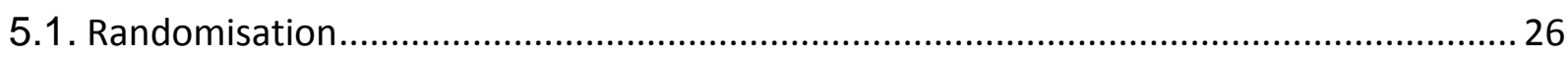

6. TREATMENT ALLOCATIONS AND PREGNANCY MANAGEMENT .......................................... 26

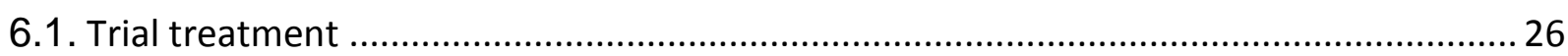

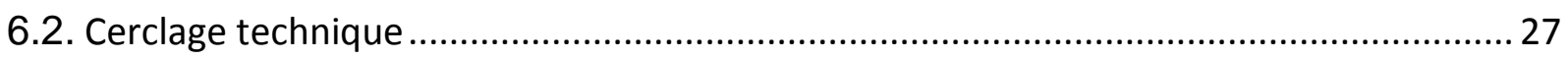

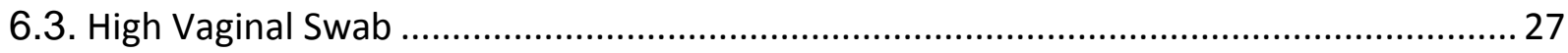

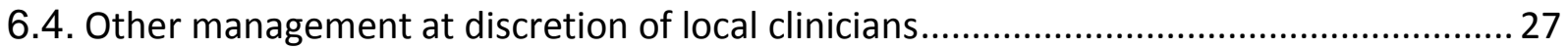

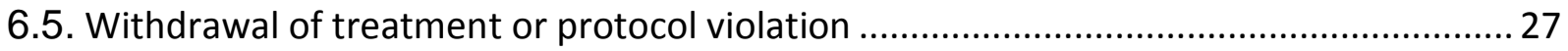

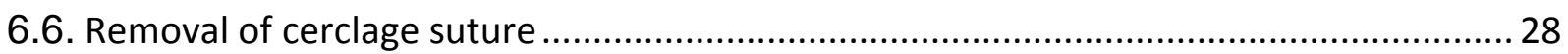

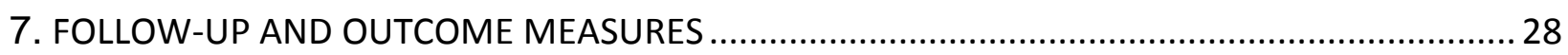

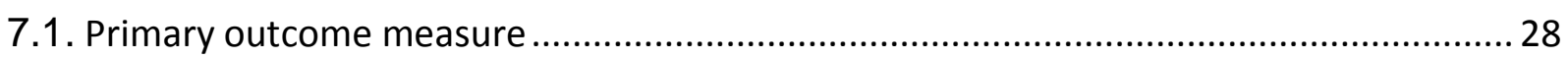

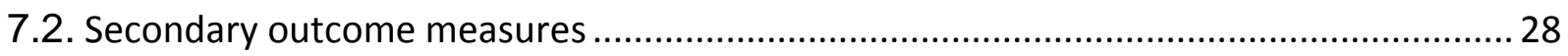

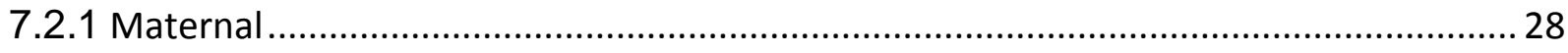

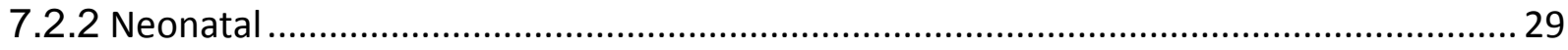

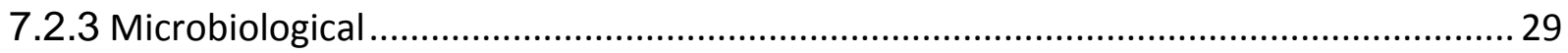

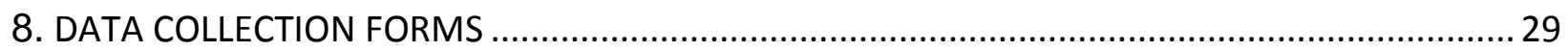

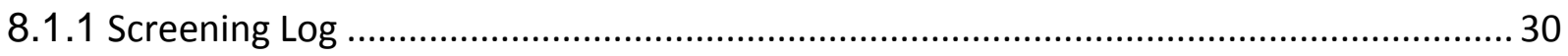

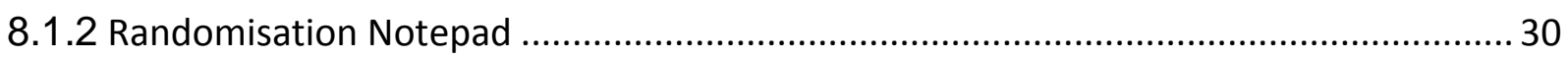

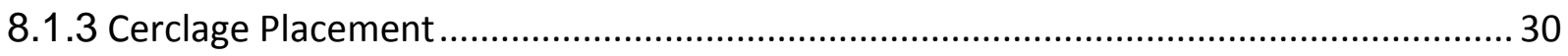

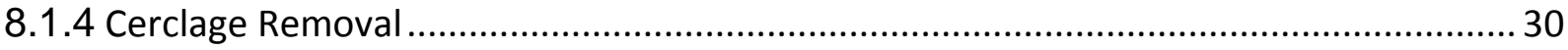

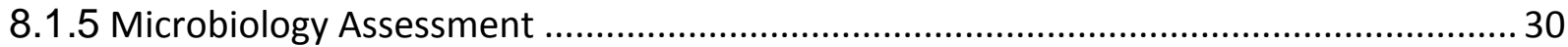

8.1.6 Pregnancy Outcome and Maternity Outcome ............................................................. 30

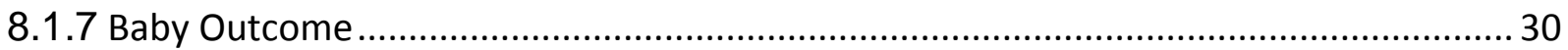

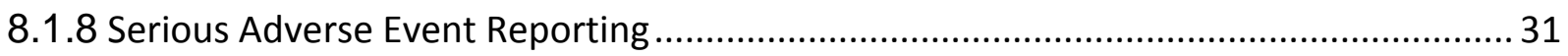

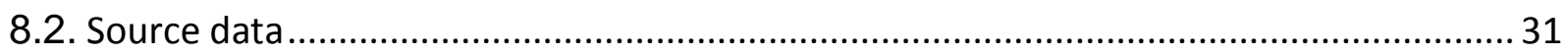

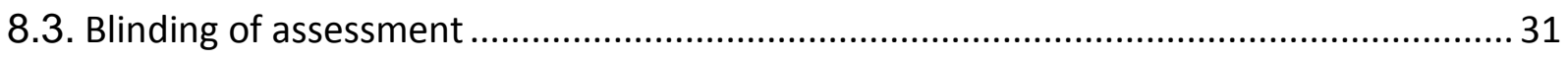

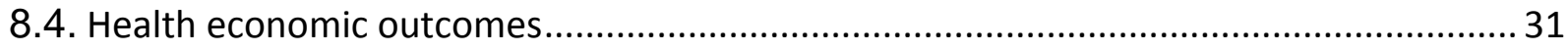

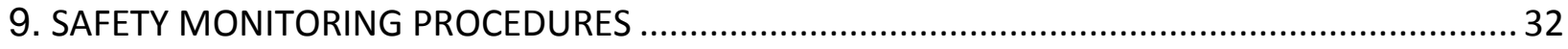

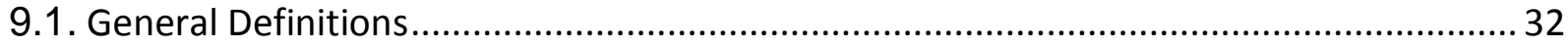

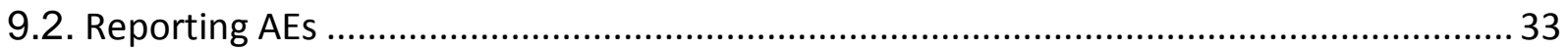

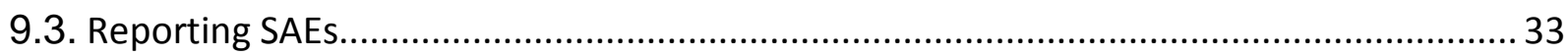

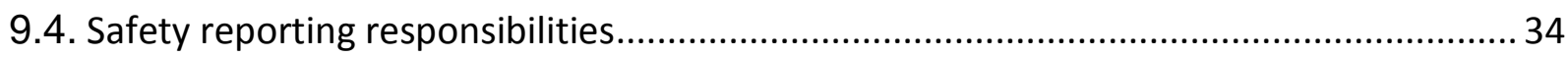




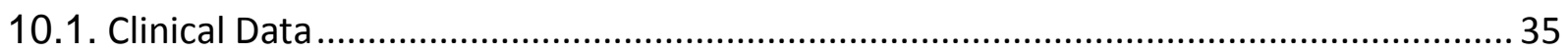

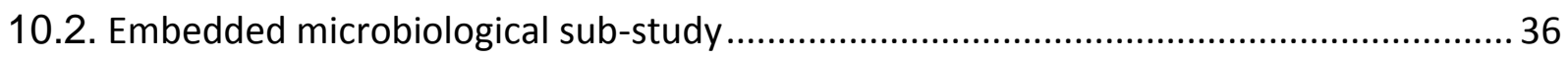

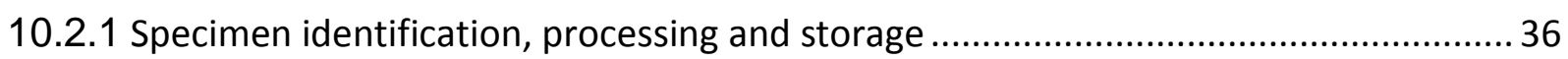

10.2.2 Quality Assurance of Microbiological Assessments.................................................. 36

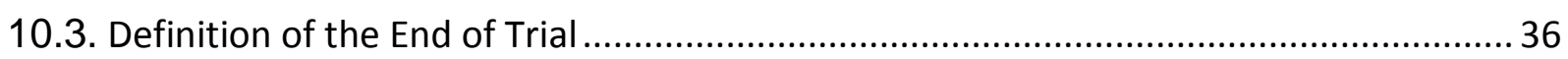

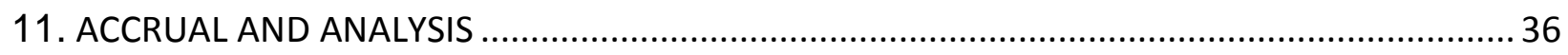

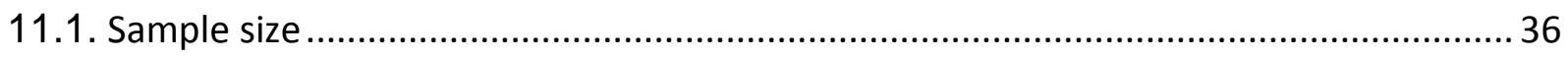

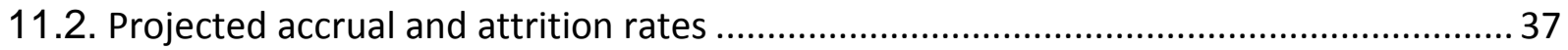

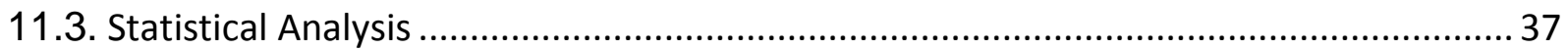

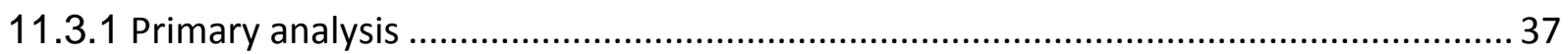

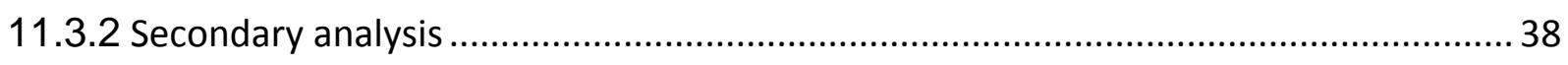

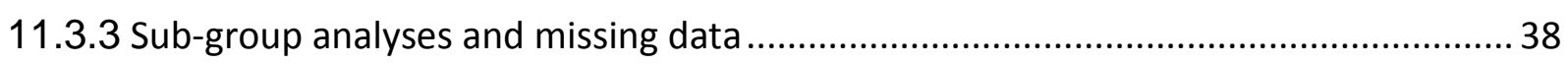

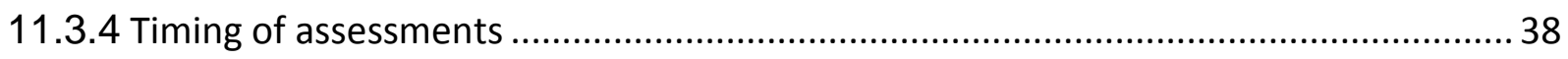

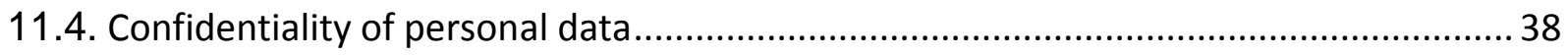

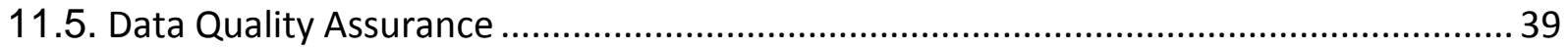

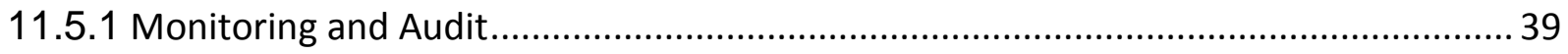

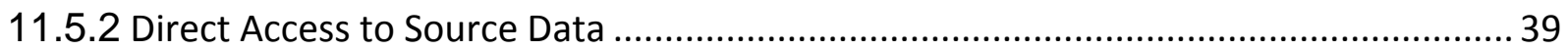

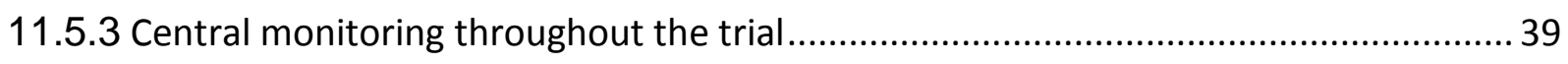

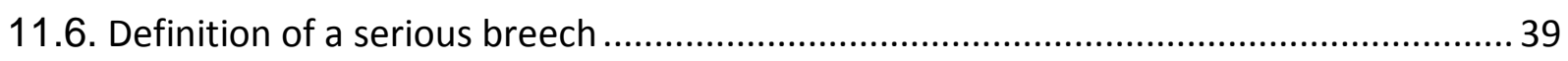

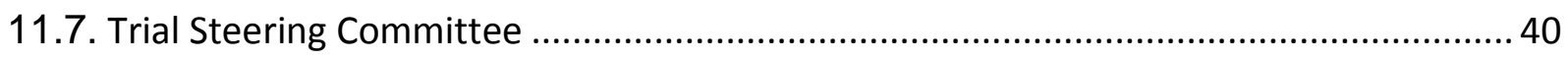

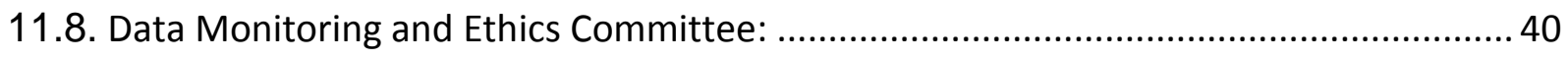

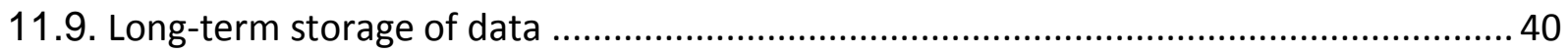

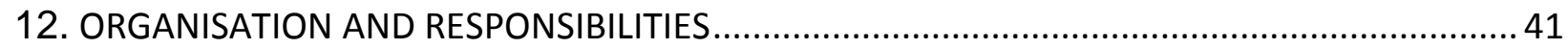

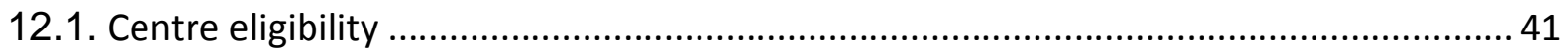

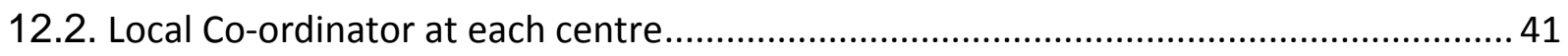

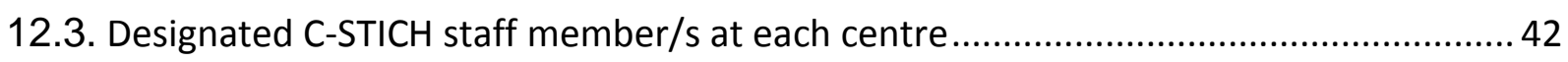

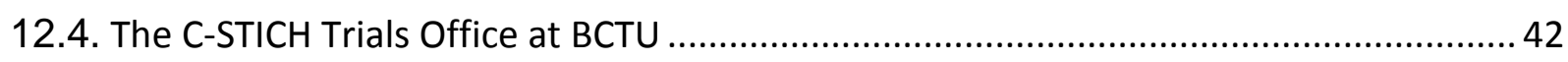

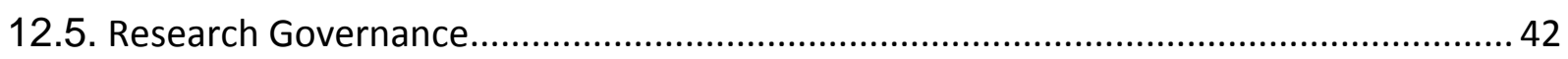

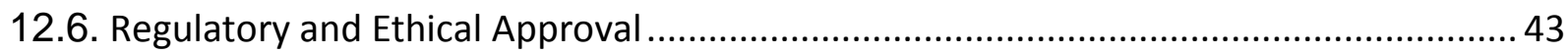

12.6.1 Ethical and Trust Management Approval or Confirmation of Capacity and Capability 43

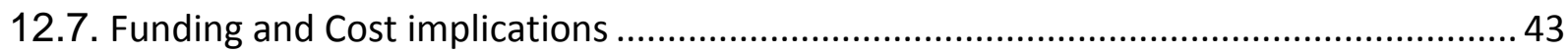

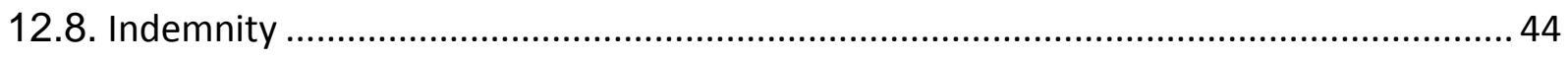

13. REPORTING, PUBLICATIONS AND NOTIFICATION OF RESULTS ........................................ 44 


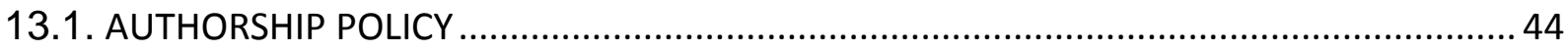

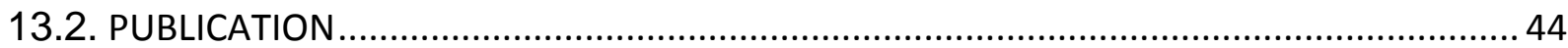

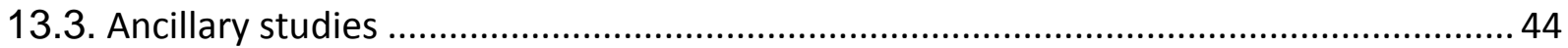

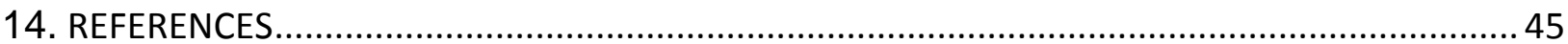




\section{BACKGROUND}

\subsection{Preterm birth}

Pre-term birth (PTB) is one of the major challenges in obstetrics and neonatology globally. According to the Office for National Statistics, approximately 50,000 babies $^{1}$ are born prematurely each year. Approximately 1,500 of them die.

An early birth puts survivors at risk of serious long-term disabilities ${ }^{2}$ and these outcomes pose a significant burden on parents as well as having economic implications on health services. It is estimated that $10 \%$ of healthcare resources in developed countries are spent on treating diseases in children resulting from PTB $^{3}$. Cervical insufficiency (also called cervical incompetence) is one of the important causes of PTB for which cerclage has been one of the established management options $^{4,5}$, and 6 .

\subsubsection{The consequences of pre-term birth}

The effects of PTB are often very severe and can be devastating for both the child and their parents. In the short term, preterm babies often require special care in the neonatal intensive care unit (NICU). In general, the earlier the preterm infant, the greater the likelihood that life supports will be required, meaning a longer stay in the NICU.

Babies born prematurely often have underdeveloped lungs meaning breathing problems are common in preterm infants and many will require ventilator support. These babies may have breathing problems through the first year of life and an increased risk for developing asthma later.

The brain continues to develop after the time of birth. The more prematurely the baby is born the more likely it is to suffer an insult which will cause damage to the brain. This can result in the failure to attain developmental milestones or in physical disability.

In the longer term, babies born prematurely often have long-term difficulties such as:

- Behavioural and social-emotional problems

- Learning difficulties

- Increased risk of conditions such as Attention Deficit-Hyperactivity Disorder (ADHD)

- Increased risk for Sudden Infant Death Syndrome (SIDS)

These children are more likely to require early intervention and special education services. Upon reaching maturity, children born pre-term are more likely to suffer chronic diseases such as heart disease, hypertension and diabetes.

With over 50,000 babies being delivered prematurely each year in the UK, in addition to the often devastating effects on both the child and its parents, pre-term birth imposes a significant economic burden on the health care system.

\subsection{Insufficient Cervix}

The cervix is the narrow tube, usually about $2.5 \mathrm{~cm}$ long that connects the uterus and vagina. Normally the lumen of the cervix remains slightly patent to allow the exit of menses and access of semen to the uterine cavity. 
Following conception and implantation of the embryo, the cervix initially becomes more vascular and softens. The lumen becomes blocked with a mucus secretion from the endocervical glands, and the thick mucus plug acts as a protective barrier to ascending infections. During a normal pregnancy a dense mesh of collagen fibres ensures that the cervix remains firm, long and closed until late in the third trimester. At this point it usually starts to soften, efface and dilate as fluid is taken up by hydrophilic mucopolysaccharides in the interstices between the collagen bundles. As the supravaginal part of the cervix expands, the cervix shortens to prepare for labour and birth.

In some pregnant women the cervix effaces and dilates prematurely. These women may suffer a second trimester miscarriage or pre-term delivery as the uterus is unable to restrain the weight of the baby pressing on the dilated cervix.

\subsubsection{Clinical presentation}

There are no objective tests that can be done before pregnancy to reliably predict an insufficient or weak cervix. Historically, women were diagnosed clinically with cervical insufficiency after they had a history of second-trimester miscarriages or early preterm births preceded by spontaneous rupture of membranes or painless cervical dilatation with no other known cause.

More recently, regular transvaginal ultrasounds from 14 to 24 weeks of gestation can detect early cervical shortening and funnelling. As with many clinical presentations, cervical insufficiency cannot be viewed as a simple dichotomous diagnosis, but instead presents as a spectrum of risk, influenced by both the anatomy of the cervix and the processes leading to premature cervical effacement and dilatation.

At a later stage, a pelvic examination can be undertaken to see if the foetal membranes have prolapsed into the neck of the cervix.

\subsubsection{Incidence}

Approximately 3,750 pregnant women are diagnosed with an insufficient cervix in the UK each year ${ }^{1}$.

\subsubsection{Risk factors}

Women considered to be more likely to experience an insufficient uterus during pregnancy are those who have had:

- Previous cervical cerclage

- A history of two or more mid-trimester losses or pre-term deliveries

- Previous cervical surgery / treatment including cervical loop biopsies

\subsection{Current management of cervical insufficiency}

Other than cerclage, very few treatments are available for cervical insufficiency and the evidence for their use is largely anecdotal. 


\subsubsection{Progesterone supplementation}

The injection of the progesterone supplement hydroxyprogesterone caproate (Makena) during the second trimester has been suggested to help women who have cervical insufficiency, although the use of this pharmaceutical is contraindicated in women carrying multiple babies.

\subsubsection{Cervical pessaries}

Some clinicians promote the use of a device which sits inside the vagina and which helps reduce the pressure on the cervix. No reliable evidence is available to determine if the use of these devices is an effective treatment for cervical insufficiency.

\subsubsection{Cervical Cerclage}

Cervical cerclage is the placement of stitches in the cervix to hold it closed and has been described as "a history-indicated suture performed as a prophylactic measure in asymptomatic women and normally inserted electively at 12-14 weeks of gestation ${ }^{8 "}$. Perhaps its most common use is in the treatment for an insufficient cervix. During this procedure, which has been in use for over one hundred years, strong sutures are placed in the lumen of the cervix to hold it closed.

Alternatively, women considered at risk can be offered ultrasound surveillance of their cervix. Insertion of a cerclage can be undertaken as a therapeutic measure in cases of cervical length shortening seen on transvaginal ultrasound. Ultrasound-indicated cerclage is performed on asymptomatic women who do not have exposed fetal membranes in their vagina. Sonographic assessment of the cervix is usually performed between 14 and 24 weeks of gestation.

There are two types of cerclage performed vaginally: McDonald's ${ }^{6}$ or Shirodkar's ${ }^{4}$. Depending on the technique used the suture may be removed shortly before the patient is ready to deliver.

- McDonald's cerclage is the most common, and is essentially a purse string stitch used to pinch the cervix shut; the cervix stitching involves placing a suture material at the upper part of the cervix while the lower part has already started to efface. This cerclage is usually placed between 12 weeks and 14 weeks of pregnancy. The stitch is generally removed around the 37 th week of gestation.

- Shirodkar's cerclage is very similar, but the sutures pass through the walls of the cervix so they are not exposed. As Shirodkar's cerclage includes a bladder dissection it is technically more difficult than McDonald's method, but is thought (though not proven) to reduce the risk of infection. The Shirodkar procedure sometimes involves a permanent stitch around the cervix which will not be removed and therefore a Caesarean section will be necessary to deliver the baby.

\subsection{The effectiveness for cervical cerclage}

\subsubsection{The evidence for cervical cerclage}

Despite being in use for over one hundred years there is very little evidence drawn from high quality clinical trials. A recent Cochrane review concluded that cerclage reduces the incidence of early delivery but does not significantly reduce miscarriage rate or perinatal mortality. ${ }^{9}$ 
Figure 1 Results of the Cochrane review and meta-analysis of RCTs of cerclage for preventing pre-term birth.

Analysis I.I. Comparison I Cerclage vs no cerclage, Outcome I All perinatal losses.

\begin{tabular}{|c|c|c|c|c|c|}
\hline \multicolumn{6}{|c|}{ Comparison: I Cerclage vs no cerclage } \\
\hline \multicolumn{6}{|c|}{ Outcome: | All perinatal losses } \\
\hline \multirow[t]{2}{*}{ Study or subgroup } & Experimental & Control & Risk Ratio & \multirow[t]{2}{*}{ Weight } & \multirow{2}{*}{$\begin{array}{r}\text { Risk Ratio } \\
\text { M-H,Fixed,95\% CI }\end{array}$} \\
\hline & $\mathrm{n} / \mathrm{N}$ & $n / N$ & M-H,Fixed,95\% Cl & & \\
\hline \multicolumn{6}{|c|}{ I History-indicated cerclage vs no cerclage } \\
\hline Ezechi 2004 & $0 / 39$ & $2 / 42$ & — & $1.9 \%$ & $0.22[0.01,434]$ \\
\hline Rush 1984 & 9/96 & 9/98 & $\leftarrow$ & $6.9 \%$ & $1.02[0.42,246]$ \\
\hline MRCIRCOG 1993 & $53 / 635$ & 661629 & \# & $51.3 \%$ & $0.80[0.56,1.12]$ \\
\hline Subtotal $(95 \% \mathrm{CI})$ & 770 & 769 & - & $60.0 \%$ & $0.80[0.58,1.10]$ \\
\hline \multicolumn{6}{|c|}{ Total events: 62 (Experimenta), 77 (Control) } \\
\hline \multicolumn{6}{|c|}{ Heterogeneity. $\mathrm{Ch}^{2}{ }^{2}=1.03, \mathrm{df}=2(\mathrm{P}=0.60) ; 1^{2}=0.0 \%$} \\
\hline \multicolumn{6}{|c|}{ Test for overall effect $Z=1.35(P=0.18)$} \\
\hline \multicolumn{6}{|c|}{2 One-off ultrasound-indicated cerclage in high risk for PTL vs no cerclage } \\
\hline To 2004 & $2 / 26$ & $3 / 30$ & $\longrightarrow$ & $22 \%$ & $0.77[0.14,4.25]$ \\
\hline Subtotal $(95 \% \mathrm{CI})$ & 26 & 30 & & $2.2 \%$ & $0.77[0.14,4.25]$ \\
\hline \multicolumn{6}{|c|}{ Total events: 2 (Experimental), 3 (Control) } \\
\hline \multicolumn{6}{|c|}{ Heterogeneity not applicable } \\
\hline \multicolumn{6}{|c|}{ Test for overall effect $Z=0.30(P=0.76)$} \\
\hline \multicolumn{6}{|c|}{3 Serial ultrasound-indicated cerclage in high risk for PTL vs no cerclage } \\
\hline Althuisius 2001 & $0 / 19$ & $3 / 16$ & - & $29 \%$ & $0.12[0.01,2.19]$ \\
\hline Berghella 2004 & $4 / 25$ & $4 / 22$ & & $3.3 \%$ & $0.88[0.25,3.11]$ \\
\hline Rust 2000 & $7 / 61$ & $5 / 66$ & - & $3.7 \%$ & $1.51[0.51,452]$ \\
\hline Owen 2009 & $13 / 148$ & $25 / 152$ & \#- & $19.1 \%$ & $0.53[0.28,1.00]$ \\
\hline Subtotal $(95 \% \mathrm{CI})$ & 253 & 256 & $\bullet$ & $29.0 \%$ & $0.66[0.41,1.06]$ \\
\hline \multicolumn{6}{|c|}{ Total events: 24 (Experimenta), 37 (Control) } \\
\hline \multicolumn{6}{|c|}{ Heterogeneity. $C \mathrm{Ch}^{2}=4.17, \mathrm{df}=3(\mathrm{P}=0.24) ; 1^{2}=28 \%$} \\
\hline \multicolumn{6}{|c|}{ Test for overall effect $Z=1.72(P=0.085)$} \\
\hline \multicolumn{6}{|c|}{4 One-off ultrasound-indicated cerclage in low/unspećfied risk for PTL vs no cerclage } \\
\hline Berghella 2004 & $0 / 3$ & or & & & Not estimable \\
\hline Rust 2000 & $5 / 43$ & $2 / 37$ & & $1.7 \%$ & $2.15[0.44,10.44]$ \\
\hline To 2004 & $7 / 101$ & 9/96 & & $7.1 \%$ & $0.74[0.29,1.91]$ \\
\hline Subtotal $(95 \% \mathrm{CI})$ & 147 & 140 & - & $8.8 \%$ & $1.01[0.46,2.22]$ \\
\hline \multicolumn{6}{|c|}{ Total events. 12 (Experimenta)), 11 (Control) } \\
\hline \multicolumn{6}{|c|}{ Heterogeneity. $C \mathrm{i}^{2}=1.30, \mathrm{df}=\mathrm{I}(\mathrm{P}=0.26) ; \mathrm{I}^{2}=23 \%$} \\
\hline Test for overall effect $Z=$ & $=0.99)$ & & & & \\
\hline
\end{tabular}

Traditionally, clinicians have used Mersilene ${ }^{\circledR}$ tape (a macroporous braided suture) for cervical cerclage because of its perceived strength and ease of removal. However, braided sutures, particularly mesh-like non-absorbable sutures, have been associated with an increased risk of infection particularly when used in potentially contaminated surgical areas ${ }^{10}$. We hypothesise that as braided sutures would have been predominant in the studies reported in the Cochrane report they may have unintentionally induced a bias in the conclusions and masked a true benefit of cerclage. 


\subsubsection{The influence of the suture material in gynaecological surgery}

Using the categorisation criteria developed at the UCLA Lichtenstein Hernia Institute ${ }^{11}$, mesh materials used in surgery are divided into four categories

\begin{tabular}{|c|c|}
\hline Category & Type of mesh \\
\hline I & Macroporous $>75 \mu \mathrm{M}$ (Atrium ${ }^{\oplus} /$ Prolene $^{\oplus}$ ) \\
\hline II & Microporous $<10 \mu \mathrm{M}\left(\right.$ Gore-Tex $^{\oplus}$ ) \\
\hline III & Macroporous with braided filaments (PTFE / Mersilene ${ }^{\circledR}$ ) \\
\hline IV & Submicronic (Silastic ${ }^{\circledR}$ ) \\
\hline
\end{tabular}

Table 1 Types of mesh

Clinically it has been reported that Type II and Type III meshes have a propensity to extrusion and infection, and there are a number of high profile products composed of Type II and Type III meshes which had to be withdrawn from incontinence and prolapse surgery due to a high erosion and infection rate ${ }^{6}$. If used in pelvic floor surgery these tapes tended to extrude into the vagina where they behaved like a wick drawing vaginal secretions up the entire course of the mesh. Microbiologically this resulted in polymicrobial infections with Gram positive and negative bacteria, anaerobes and aerobes leading to severe complications.

The difference in behaviour of meshes was demonstrated in a series of laboratory experiments in rats $^{12}$. During these experiments it was observed that the Type II and Type III meshes set up a chronic inflammatory response with the formation of giant cells. This never progressed to a stable state with fibrous deposition as one would see with a Type I mesh. As a consequence of the chronic inflammatory response the mesh soon became encapsulated with very little vascularisation thus limiting even further its ability to withstand infection.

Mersilene ${ }^{\circledR}$ (Type III mesh) has been used in a clinical study looking at repair of vaginal prolapses. In this study the patient underwent a sacrocolpopexy with the mesh being introduced either abdominally or vaginally. Results showed that the group where the tape had been introduced abdominally showed a $3 \%$ erosion rate, whilst those women in whom the tape had been introduced vaginally exhibited a $20 \%$ erosion rate. This was interpreted to show that as well as the structure of the mesh, the environment it is exposed to also has a role to play ${ }^{13}$.

A series of simple laboratory experiments demonstrated clearly that Mersilene ${ }^{\circledR}$ had very pronounced capillary and fluid absorbing properties which aided the propagation of Staphylococcus (a bacterium usually considered immobile) from an infected chamber to a sterile one. This did not occur with monofilament suture. The hypothesis therefore is that a suture constructed from Mersilene ${ }^{\circledR}$ would magnify these findings ${ }^{14,15}$. Supporting evidence for this was provided by the Dalkon shield, a contraceptive intrauterine device composed of a braided thread, which was found to cause pelvic infections in a disproportionately large percentage of its users before being withdrawn from the market ${ }^{16}$.

Most surgeons began to convert to monofilament sutures at this time ${ }^{17}$, and all of the Type II and Type III products are nowadays used rarely in a clinical setting.

The FDA has issued warnings about mesh products, especially Type II and Type III. Complications associated with the mesh insertion in incontinence and pelvic floor surgery, such as extrusion and 
infection, led to clear recommendations from Royal College of Obstetrics and Gynaecology to issue guidance on when, how and by whom the mesh should be inserted and removed ${ }^{17}$. NICE guidance supports the use of mesh in some surgical procedures provided that the normal arrangements for consent, audit and clinical governance are in place ${ }^{18}$. Nonetheless, mesh is considered to be a medical device and so any complications should be reported to Medicines and Healthcare products Regulatory Authority (MHRA).

\subsubsection{Use of braided sutures in cerclage}

There is a paucity of evidence concerning the use of Mersilene ${ }^{\circledR}$ in the context of cervical cerclage and the nature of the suture used is often determined by the surgeon's personal preference. Some surgeons opt to use a non-braided monofilament sutures for cerclage. Conversely detractors suggest that monofilament sutures are not as strong and can potentially traumatise the cervix at insertion. However, these claims are not substantiated by any scientific or clinical evidence.

With its braided nature Mersilene ${ }^{\circledR}$ is proposed to stimulate a chronic inflammatory response and the suture becomes encapsulated. This means that after the securing knot is cut, the lack of fibroblast infiltration results in the easy withdrawal of the suture from the cervical tissue ${ }^{14,15}$. However, there is some evidence that Mersilene ${ }^{\circledR}$ also has pronounced capillary and absorbent properties and so may act as a wick. This wick may provide an environment where bacteria can grow and a route by which they can migrate and enter the uterus where they may cause a chronic infective state and stimulate early labour. This suspected wicking is prompting many specialists to move towards the adoption of monofilament sutures.

However, some surgeons claim that non-braided sutures are not as strong as braided sutures and increase cervical trauma at insertion. Type I (single strand) material does not set up a chronic immune response, and at removal has been found to be incorporated tightly into its surrounding tissue. That this material has been assimilated can make it extremely difficult to remove without causing significant tearing and damage. Obstetricians and other non-urogynaecology specialists have little experience with meshes or tapes so would not have been aware of the potential for the Mersilene ${ }^{\circledR}$ tape to contribute to premature delivery.

\subsubsection{Preterm birth and infection}

One hypothesis states that preterm labour results from an increased susceptibility to infection, and it is recognised that both low-grade chronic and acute infections are risk factors for PTB.

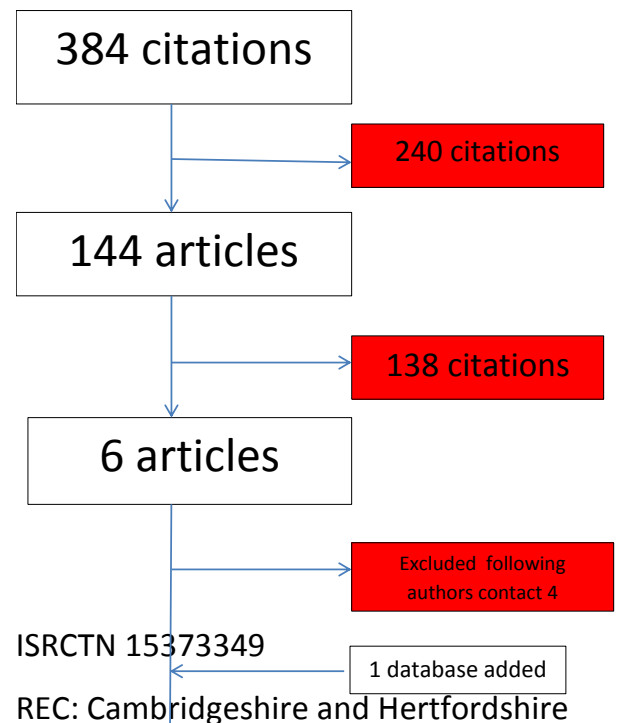

Women who suffer from an insufficient cervix may have an anatomical arrangement which increases the risk of an infective process occurring, thus predisposing them to preterm labour. In these women the use of a tape to restore mechanical integrity may inadvertently introduce a route by which an infection stimulates PTB.

\subsection{The evidence for effectiveness of the suture material used for cervical cerclage}

A comprehensive literature search of MEDLINE, EMBASE, CINHL and ISRCTN from their inception to December 2013 
identified no randomised controlled trials comparing the nature of the suture material in relation to planned/elective cerclage. Using search terms of Cerclage, cervix, suture, and Mersilene ${ }^{\circledR}$ suture, the search was extended to non-randomised studies (NRS). Figure 2. Initially, only two published studies were identified to which data has subsequently been supplemented with unpublished data from Professor Bennett (personal communication). The outcomes considered was pregnancy loss, which included miscarriage and neonatal death.

The NRS meta-analysis demonstrates that non-braided sutures, compared to braided, were associated with a pregnancy loss rate of $7 \%$ compared to $19 \%$ respectively (relative risk was 0.34 [95\% $\mathrm{Cl} 0.18$ to 0.63$]$ ). Figure 3.

Figure 2 Identification and selection of studies for meta-analysis

Figure 3 Non-randomised study meta-analysis of pregnancy loss

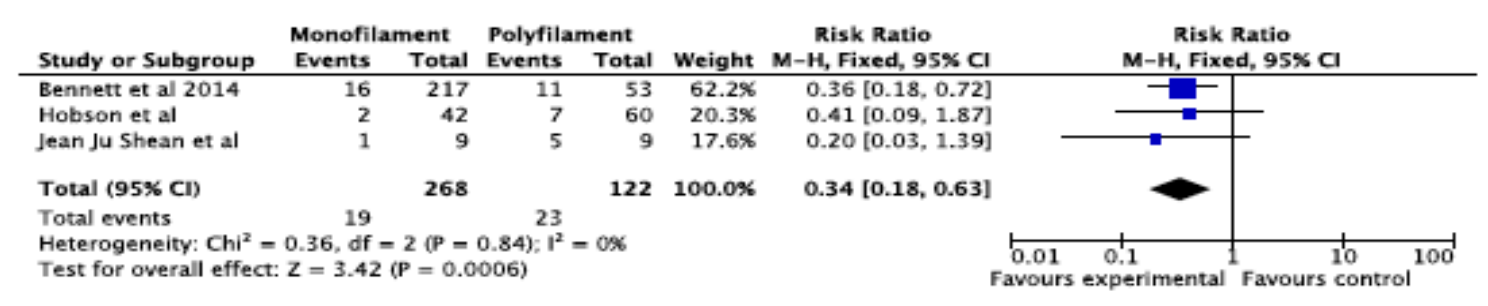

\subsection{The choice of questions to be asked}

\subsubsection{Rationale}

To date, the effectiveness of the cerclage procedure in the prevention of pregnancy loss remains uncertain ${ }^{11,16}$. The lack of clear and widely accepted criteria for inserting a cerclage is a possible reason for the inability of the current literature to demonstrate a clinical benefit of cerclage procedures.

Another potential confounding factor, and the hypothesis on which the C-STICH trial is based, relates to the nature of the suture material used. As they were traditionally used at the time it is not unreasonable to assume that the RCTs that provide the data for the Cochrane review used braided sutures to place a cerclage. If these procedures were performed using a monofilament suture material and the difference in foetal survival identified in our retrospective cohort study between monofilament and braided is true, cervical cerclage may be shown to be a significantly effective procedure.

\section{STUDY OBJECTIVE}

\subsection{Primary objective}

To examine the effect of using monofilament suture material compared with braided suture material on pregnancy loss rate in women deemed to be at risk of an insufficient cervix and treated with cervical cerclage

\subsection{Secondary objectives}

- To assess the effect of suture material on other pregnancy and neonatal outcomes

- To explore the variation in effect between McDonald's and Shirodkar's cerclage, especially with reference to bladder dissection 
- To explore the variation in effect between the indication for cerclage

- To produce advice and a video clip to illustrate best practice in cerclage stitch insertion and removal

\section{TRIAL DESIGN}

\subsection{Design}

C-STICH is a multicentre, open, randomised controlled trial.

\subsection{Setting}

The C-STICH study will run in at least 32 NHS obstetric and gynaecology units in the UK and Ireland. Recruitment will be in antenatal clinics, outpatient departments, and obstetric and gynaecology wards. Randomisation and insertion of the cerclage will be performed in antenatal clinics, day surgery areas, surgical in-patient settings and delivery suites.

\section{STUDY POPULATION}

The target population for C-STICH are women attending antenatal clinics or admitted to obstetric and gynaecology wards in whom the reviewing clinician believes that the placement of a cervical cerclage is the most appropriate method to prevent a miscarriage or pre-term birth.

These women will be invited to take part in the C-STICH trial at the time the decision is made to undertake a cervical cerclage procedure providing that they meet the eligibility criteria below.

\subsection{Eligibility Criteria}

\subsubsection{Inclusion}

- Singleton pregnancy

- Indication for cervical cerclage for either:

- A history of three or more previous midterm losses or premature births ( $\leq$ 28 weeks), OR

- Insertion of cervical sutures in previous pregnancies, $O R$

- A history of midtrimester loss or premature birth with a shortened $(\leq 25$ $\mathrm{mm}$ ) cervix, OR

- Women whom clinicians deem to be at risk of preterm birth either by history or the results of an ultrasound scan

\subsubsection{Exclusion}

- Women who have taken part in C-STICH previously

- Women aged less than 18 years old at the time of presentation

- Those with a multiple pregnancy

- Those requiring a rescue cerclage *

- Women who are unwilling or unable to give informed consent

- Those in whom a cerclage will be placed by any route other than vaginally (e.g. via an abdominal route) 
- Immediate need for insertion of a suture**

- Women who have membranes that have ruptured or are surfacing***

* For study purposes, rescue cerclage is defined as: emergency cerclage where stitches are inserted in women who have had their preterm labours (e.g. uterine contractions, progressive cervical dilatation, bulging membranes) sufficiently halted by tocolysis or other means between 15 and 28 weeks.

** Immediate need for insertion of a suture should not be delayed by the trial (thus, if giving information about the trial and waiting for the participant to decide upon whether or not she wants to participate will delay the insertion of an urgently needed suture, then treatment should go ahead and the woman should be excluded from the trial).

***Woman with membranes that are ruptured or bulging through the external OS should have a rescue cerclage and be excluded from trial participation.

\subsection{Identifying potential participants}

Potentially eligible women will be identified in antenatal clinics, in obstetric and gynaecology wards, in antenatal clinics, or outpatient departments, and invited to join the trial at the time the decision is made to undertake a cervical cerclage.

\subsection{Approaching potential participants for consent}

Potential participants will only be approached by suitably qualified and experienced personnel whose names appear on the delegation log.

\subsubsection{Obtaining consent}

All women who are referred to secondary care for cervical cerclage should be considered potentially eligible and should be screened prior to their antenatal appointment by the designated C-STICH staff member in each centre. The obstetrician or gynaecologist who will be providing the woman's clinical care and performing the procedure will discuss preventative options and establish eligibility based on history and preferences.

The indication for the cerclage should be discussed before the trial is introduced. Adequate time should be allowed for consideration of participation in the trial before the cerclage procedure is undertaken. There is no pre-agreed specified time to consent. Consent should be informed and voluntary with time for questions and reflection. However, the patient has the right also to make an immediate decision to consent.

Consent to participate in C-STICH will be sought by appropriately qualified staff who are both GCP trained and designated by the Principal Investigator to conduct informed consent procedures. Women will be asked to confirm their consent to participate in the C-STICH trial by initialling the appropriate boxes on the consent form and signing the form in the presence of the person taking consent. A copy should be given to the women; one to be kept in the patient notes, the original should be kept in the local site file and one copy sent to the C-STICH Trial Office.

The trial site will be asked to complete the Baby Outcome form to provide information on neonatal mortality. Where this information is unavailable from the site, because for example, the mother has delivered at a site other than the recruiting site, it will be sought from the site at which the mother delivered her baby. If this site is not participating in $\mathrm{C}-\mathrm{STICH}$, permission will be sought from the site to add them to C-STICH as a continuing care site. If is unknown as to where the 
mother has delivered her baby, the mother's GP will be contacted and asked to provide the details.

Any data which is unobtainable from either the continuing care site or the recruiting site will be sought from the NHS Digital (England and Wales) or ISD Scotland). NHS numbers assigned to the participant's babies will be passed on to NHS Digital or ISD Scotland with a view to obtaining any corresponding death information. The participant will be made aware of our intentions to request data from the continuing care site and / or NHS Digital or ISD Scotland in the C-STICH Participant Information Sheet and their agreement will be recorded on the consent form.

All women approached for study participation should be recorded anonymously on the screening $\log$, available in the investigator site file, which should be passed to the coordinating centre in an anonymised form.

\subsubsection{Informing the participant's GP}

Following the participant granting consent, her GP should be notified using the study template "Letter to GP".

\subsection{Ineligible patients}

If a woman is screened but is not eligible for the trial, be it due to a preference for the use of a particular suture type, a contraindication, a pathological reason, or consent for randomisation is not given, an anonymous record of the case should be kept in the screening log. The screening log will collect initials, age group, ethnic group, and the reason each patient is not eligible to participate in the trial. Women who consent and are subsequently found to be ineligible should also be noted.

The screening log should be kept in the site file and a copy sent to the C-STICH Trial Office on a monthly basis. The members of the trial co-ordination team will be unable to identify women based on the information provided. This screening log information will inform updates to the funder regarding recruitment targets for C-STICH Trial.

\subsection{CO-ENROLMENT}

Women already participating in another trial, whether it be observational or an IMP can be enrolled into C-STICH.

After a woman is randomised to the C-STICH trial she can participate in further trials of investigational medicinal products (IMPs), although IMP trials for the prevention of second trimester miscarriage or pre term birth or any trials with the same primary outcome should be excluded (such as trials of tocolytic agents).

Women participating in C-STICH can be recruited into observational studies for the prediction or diagnosis of PTB or premature labour.

\section{RANDOMISATION}

The participant should be randomised just prior to the cervical cerclage procedure, to minimise the number of withdrawals and protocol violations, but allowing sufficient time for the obstetrician to prepare the sutures for the procedure. 


\subsection{Randomisation}

Birmingham Clinical Trials Unit will provide a bespoke web-based randomisation with telephone back-up. Patients are entered and randomised into the trial by logging into secure online webpage available at www.birmingham.ac.uk/C-STICH. Each person eligible to randomise will be provided with a unique username and password. The online randomisation is available 24 hours a day, 7 days a week apart from short periods of scheduled maintenance and when there are occasional network interruptions. Alternatively, investigators can make a Freephone telephone call (Tel $08009530274)$ to the randomisation service. This telephone randomisation service is available between $0900-1700$ hrs Monday to Friday.

Randomisation Forms will be provided to investigators and should be used to collate the necessary information prior to randomisation. All questions and data items on the Randomisation Form will need to be answered before a trial number and allocation can be given. If an essential data item is missing, randomisation will be suspended but can be resumed once the information is available. Only when all eligibility criteria and baseline data items have been provided will a trial number and treatment allocation be given. This will be followed by a confirmatory email sent to the randomising person, local Principal Investigator and the main research staff member within the centre.

Participants will be randomised at the level of the individual in a 1:1 ratio. A minimisation procedure using a computer-based algorithm will be used to avoid chance imbalances in treatment allocation and the following potentially important variables:

- Indication for the cerclage (history / ultrasound), thus:

- A history of three or more previous midterm losses or premature births $(\leq 28$ weeks), OR

- Insertion of cervical sutures in previous pregnancies, OR

- A history of mid trimester loss or premature birth with a (current) shortened ( $\leq 25$ $\mathrm{mm}$ ) cervix, OR

- Women whom clinicians deem to be at risk of preterm birth either by history or the results of an ultrasound scan.

- Technique planned (with or without bladder dissection)

- Intention to commence patient on progesterone (yes / no)

- Randomising centre

A 'random element' will be included in the minimisation algorithm, so that each patient has a probability (unspecified here), of being randomised to the opposite treatment that they would have otherwise received. Full details of the randomisation specification will be stored in a confidential document at BCTU.

\section{TREATMENT ALLOCATIONS AND PREGNANCY MANAGEMENT}

\subsection{Trial treatment}

Depending on the results of the randomisation, cerclage will be performed with either a monofilament or braided suture. Both types of suture used in C-STICH are standard surgical 
materials already in use. However, there are different brands of each suture material and whilst the trial does not specify which brand a surgeon should use, the use of Mersilene ${ }^{\circledR}$ and Ethilon is encouraged in order to standardise sutures as far as possible. The suture material used must be CE marked for this purpose. The most commonly used are Mersilene ${ }^{\circledR}$ a nonabsorbable, braided, sterile surgical suture composed of poly-ethylene terephthalate and Ethilon, a nonabsorbable, monofilament, sterile surgical suture composed of the long-chain aliphatic polymers Nylon 6 and Nylon 6,6.

The MHRA have confirmed that C-STICH is not classed as a device trial.

\subsection{Cerclage technique}

The technique of suture insertion (i.e. with or without bladder dissection) will be at the surgeon's discretion and may include an additional occlusion stitch to close the external os. Should an additional occlusion stitch be used we request that the same suture type as the randomised allocation be used. To ensure the groups are balanced as evenly as possible, the proposed cerclage technique will be taken into account in the allocation of the type of suture used, so this must be decided before randomisation.

\subsection{High Vaginal Swab}

Where possible, a high vaginal swab should be taken at the time the cerclage is placed. Ideally the HVS should be taken in theatre prior to any vaginal cleaning and preparation or antibiotic use. If this is not possible, a HVS can be taken before cerclage insertion but only if the participant is not taking any concomitant antibiotics. Additionally, where a swab has been taken, the swab should be sent to the local microbiology department, where a Gram stain and aerobic and anaerobic culture should be undertaken. The presence or absence of a panel of potentially pathogenic microorganisms should be recorded on Case Report Form (CRF) 4, Microbiology Assessment. Microbiology Departments should follow their local SOPs when issuing clinical reports of results.

\subsection{Other management at discretion of local clinicians}

Apart from the trial treatments allocated at randomisation, all other aspects of PTB prevention management e.g. progesterone will be at the discretion of the care-providing clinician.

In rare instances, a second cerclage stich is required later in the pregnancy. In this case, the participant cannot be randomised a second time but should ideally be sutured with the same suture material as used in the initial cerclage. Please note, when placing additional sutures we do not request that you take a second high vaginal swab. All subsequent cerclages will be recorded on CRF 2 and CRF3.

The pregnancy should be managed as per current usual practice for women with a cerclage in situ, with no other special treatments, no special investigations, and no extra follow-up visits outside those required clinically.

\subsection{Withdrawal of treatment or protocol violation}

Whilst a participant may voluntarily withdraw from this study at any time, it is impossible to change the allocated treatment once the cerclage procedure has been performed, as it would be unsafe and unethical to remove and replace the suture thread. 
Unless withdrawn from the study, if a participant does not return for a standard antenatal appointment, attempts will be made to contact her to collect pregnancy outcomes and adverse events. If a woman decides, after randomisation, she does not wish to have the cerclage, or the randomly allocated suture, she may withdraw herself from the trial treatment. The timing of randomisation as close as possible to the procedure should minimise the number of postrandomisation withdrawals or violations.

Clear distinction will be made as to whether a participant is withdrawing from the trial but will still be followed up on an intention-to-treat basis, or whether the participant refuses any follow-up. If a participant explicitly withdraws consent to have any further data recorded their decision will be respected and recorded. All communication surrounding the withdrawal will be noted in the patient's hospital records and trial database, and no further data will be collected for that participant.

Should a woman lose capacity to provide continued consent, they will be assumed to wish to remain in the $\mathrm{C}-\mathrm{STICH}$ trial as there would be no further procedures or tests required for the trial.

\subsection{Removal of cerclage suture}

Planned removal of the suture would occur at 37 ( \pm 1 week) weeks' gestation and the suture will be removed by the method the clinician feels most appropriate.

Where possible, upon removal, the suture should be sent to the local microbiology laboratory for aerobic and anaerobic culture. Doing the microbiology assessment is optional, however, where this has been done, the presence or absence of a panel of potentially pathogenic microorganisms should be recorded on CRF 4. Most Microbiology laboratories would not routinely process suture material, and we do not expect that it will be necessary to issue a clinical report of the culture result. Where a local laboratory chooses to do so, they should follow their local SOP for reporting of results.

\section{FOLLOW-UP AND OUTCOME MEASURES}

\subsection{Primary outcome measure}

- Pregnancy loss rate (miscarriage and perinatal mortality, including any still birth or neonatal death in the first week of life)

\subsection{Secondary outcome measures}

\subsubsection{Maternal}

- Time from conception to pregnancy end (any reason)

- Miscarriage \& pre viable neonatal death (defined as delivery $<24$ weeks)

- Stillbirth (defined as interuterine death $>=24$ weeks)

- Gestation at delivery (in live births $>=24$ weeks)

- Gestational age $<28 /<32 /<37$ weeks at delivery (in live births $>=24$ weeks)

- Time from conception to onset of spontaneous vaginal delivery (in live births $>=24$ weeks)

- Sepsis (at any time in pregnancy and until 7 days postnatal)

- Preterm pre labour rupture of membranes (PPROM) 
- Gestational age at PPROM

- Mode of initiation of labour (spontaneous or induced)

- Mode of delivery (vaginal or operative vaginal or caesarean)

- Cerclage placement complications (cervical laceration/bleeding from cervix/ruptured membranes/bladder injury)

- Cerclage removal complications (cervical tears/need for anaesthetic/difficult to remove)

- Other maternal complications: vaginal bleeding/steroid use/chorioamnionitis/maternal pyrexia of $38^{\circ} \mathrm{C}$ (intrapartum/postnatal)/systemic infection requiring antibiotics (intrapartum/postnatal)/admission to HDU or ITU (pre/post-delivery)

- Serious adverse events

\subsubsection{Neonatal}

- Early neonatal death (defined as a death within 7 days after delivery)

- Late neonatal death (defined as a death beyond 7 days and before 28 days after delivery)

- Birth weight adjusted for gestational age and sex (in live births $>=24$ weeks)

- Small for gestational age and sex (<10th centile; in live births $>=24$ weeks)

- Resuscitation at birth/additional care required (SCBU/NICU/HDU/transitional)/length of stay in additional care

- Antibiotics within 72 hours/sepsis (clinically diagnosed/proven)

- Early neurodevelopmental morbidity (severe abnormality on cranial ultrasound scan)

- Respiratory support (ventilation/CPAP)/days on respiratory support/supplementary oxygen requirements at 36 weeks post menstrual age

- Necrotising enterocolitis (Bell's stage 2 or 3)

- Retinopathy of prematurity requiring laser treatment/disabilities/congenital abnormalities

- Serious adverse events

\subsubsection{Microbiological}

Full cultures will be undertaken to identify the complete range of potentially pathogenic bacteria isolated from the suture, and high vaginal area. The likely significance of microorganisms isolated from each clinical sample will be assessed in the context of clinical evidence of infection in the mother and her baby.

\section{DATA COLLECTION FORMS}

Data for the purpose of assessing the efficacy and safety within the C-STICH trial will be collected from the clinical team responsible for the participants care on a number of data collection (case report) forms (CRFs). Data required for the primary and the majority of secondary outcomes are objective measures which are routinely collected for clinical purposes and will be transcribed from patient records. 
The CRFs should be completed either in paper form and either, be returned to the C-STICH Trial office to be entered onto the database, or they can be entered directly onto the online database by those with access. The patient's NHS and hospital number will be collected and both may be used in the process of collecting missing data.

\subsubsection{Screening Log}

The Screening Log is designed to collect basic details of all women approached, including those who are found to be ineligible and those that decline their invitation to participate. We do not expect, nor should there be any record, of patients on the log who are not at risk of pre-term birth or who are not indicated to have a cervical cerclage, i.e., are indicated for pessaries or pessaries and cervical length scans. All patients indicated on the log therefore should be only those at risk of pre-term birth who are indicated for cerclage. Information should be added as to the Trial Number (TNO) if randomised, the reason for ineligibility where relevant, e.g., patient requires a rescue cerclage or has ruptured membranes, or give the reason for declining where you have this. The screening log should be kept up to date by the designated C-STICH staff member.

\subsubsection{Randomisation Notepad}

At the first clinic visit, the gynaecological and obstetric clinical history of the woman will be taken. Details of ultrasonographic assessments will be collected alongside basic demographic details. Key prognostic details should be recorded on the Randomisation Notepad (CRF 1) prior to randomisation as these will be needed for minimisation. This form also contains a checklist for eligibility.

\subsubsection{Cerclage Placement}

The local approved designated C-STICH staff member should report details of the cerclage placement procedure using the Cerclage Placement Form (CRF 2), providing information to include: the taking of a microbiological swab, use of and type of antisepsis, technique used, the suture thread used, the number of "bites", the position of any knots, and use of any tocolytic agent.

\subsubsection{Cerclage Removal}

Please use the Cerclage Removal form (CRF 3) to record details of the removal of sutures. The Cerclage Removal form has been designed to also collect information about further cerclages that have been placed since placement of the initial randomly allocated cerclage.

\subsubsection{Microbiology Assessment}

The Microbiology Assessment form (CRF 4) should be used to record outcomes of microbiology assessments done on any swabs and suture thread/s taken. Please use a separate case report form to record microbiology outcomes for either the high vaginal swab or the removed suture/s.

\subsubsection{Pregnancy Outcome and Maternity Outcome}

The Pregnancy Outcome and Maternity Outcome forms (CRF 5, PART A and PART B) should be used to record the primary outcome and secondary maternal outcomes at the conclusion of the pregnancy.

\subsubsection{Baby Outcome}

Please use the Baby Outcome form (CRF 6) to record information about the baby's status. Only complete this form where you recorded a 'live birth' on CRF 5, PART A, the Pregnancy Outcome form. 
The end of neonatal follow up will be different for neonates born preterm compared with term. For preterm neonates (born less than 37 weeks), the end of trial follow-up will be the estimated date of delivery (as confirmed by first trimester ultrasound) or discharge from hospital whichever occurred sooner.

For term neonates (born after 37 weeks), the end of trial follow up will be 28 days post-delivery or discharge from hospital whichever occurred sooner.

The baby outcome form should be completed at either of the above time points or at discharge from hospital whichever is soonest.

Where information regarding the baby's status upon discharge from hospital or at the above time points is not available from a site, the Trial Office will attempt to obtain it from the continuing care site where relevant or NHS Digital or ISD Scotland using the babies NHS / CHI number, as described in Section 4.3.1 above. To ensure complete collection of late perinatal mortality the trial will request perinatal mortality data for all babies discharged from hospital before 28 days postnatal from the continuing care site or NHS Digital or ISD Scotland.

\subsubsection{Serious Adverse Event Reporting}

The Serious Adverse Event Reporting Form (CRF 7) should be used to record details of SAEs that are defined and described in Section 9 below. The 'initial' SAE report should be followed up by a detailed 'follow-up' report as soon as further information becomes available.

\subsection{Source data}

For the purposes of the C-STICH trial, source data comprises of:

- Clinical notes (paper and electronic)

- High vaginal swabs and the removed sutures or other material used for microbiological analysis.

\subsection{Blinding of assessment}

Clinicians cannot be blinded to the allocation, and will need to document the details of the cerclage procedure in the operative notes. Should suture allocation need to be recorded in the hospital notes to facilitate the placement of the correct randomised suture (e.g. as cerclage is to be performed later by a different clinician) this is acceptable. The patient, microbiologists, neonatologists and other members of the clinical team responsible for the woman's care should be blinded to the allocation as far as feasible and safe.

\subsection{Health economic outcomes}

In view of the similarity in prices of suture material used in the trial, the fact that the surgical procedure used for inserting both suture materials is identical, and the high cost of care of preterm babies, any difference in pregnancy loss rate is going to dominate an economic evaluation. However, the primary and secondary outcomes collected will provide enough information to assess any potential cost saving of one type of suture material over the other if a difference is identified. 


\section{SAFETY MONITORING PROCEDURES}

There may be unexpected serious adverse reactions associated with monofilament or braided sutures when used in cervical cerclage. Monofilament or braided sutures have been used to treat cervical cerclage for many years and there is no reason to believe there are adverse biochemical reactions intrinsic to the material of the suture thread, but there may be adverse events arising from the biomechanical properties of the thread. There are also known adverse events of cerclage irrespective of suture material used.

This protocol distinguishes adverse events from outcomes.

It is the responsibility of investigators to notify serious adverse events to the C-STICH Trial Office, who will forward these to the sponsor. It is the remit of the sponsor to report to the ethics committee. It is therefore imperative that all investigators have a thorough understanding of anticipated serious adverse events and the reporting process of these events.

\subsection{General Definitions}

\section{Adverse Events (AE)}

An $A E$ is:

- Any unintentional, unfavourable clinical sign or symptom such as:

- Any new illness or infection or the deterioration of existing disease or illness

- Any clinically relevant deterioration in any laboratory assessments or clinical tests, for example continued shortening of the cervix or dilatation.

AEs do not need to be reported unless they fulfil the condition of an SAE as below:

\section{Serious Adverse Events (SAEs)}

An SAE is an untoward event which:

- Results in death*

- Immediately threatens the life of participant**

- Results in hospitalisation or a longer than anticipated stay in hospital

- Results in a persistent or significant disability

- Is an important medical event that may jeopardise the patient or requires medical intervention to prevent one of the outcomes above.

- Congenital malformations, abnormalities identified on the mid trimester scan will not be recorded as SAEs. Only congenital abnormalities first identified in the neonatal period should be considered SAEs.

*All maternal deaths will be reported to BCTU on the SAE Form irrespective of whether the death is related to pregnancy, the cerclage procedure, or an unrelated event. If a participant dies, any post-mortem findings must be provided to BCTU. BCTU will report all deaths to the DMEC, chief investigator and sponsor for continuous safety review. 
** Life-threatening in the definition of a serious adverse event refers to an event in which the mother was at risk of death at the time of the event. It does not refer to an event which hypothetically might have caused death if it were more severe. Important adverse events that are not immediately life-threatening or do not result in death or hospitalisation, but may jeopardise the pregnancy or may require intervention to prevent one of the other outcomes listed in the definition above, should also be considered serious.

\section{Expected SAEs}

Expected SAEs also include, but are not limited to, the following:

- Premature rupture of membranes within 48 hours of the procedure

- Infection of the amniotic sac (chorioamnionitis) requiring intravenous antibiotics.

- Preterm labour or miscarriage within 48 hours of cerclage

- Other conditions threatening the life of the mother

- Severe cervical lacerations at time of procedure

- Bladder injury as a result of the cerclage procedure

A miscarriage, preterm delivery or neonatal death 48 hours after the cerclage procedure will be considered an outcome and not an adverse event, and should be reported according to Section 8.1.7 (Pregnancy outcome form). Miscarriages, still births or neonatal deaths within 48 hours of the cerclage procedure should also be reported as SAEs.

Events NOT considered to be SAEs, and therefore should not be reported as such, are hospitalisations for:

- routine monitoring or removal of cervical cerclage more than 48 hours after the procedure

- treatment, which was elective or pre-planned, for a pre-existing condition that is unrelated to the pregnancy

- admission to a hospital for delivery of the baby

- management of a premature baby

\subsection{Reporting AEs}

Although non-serious adverse reactions or events are not required to be reported to the Trials Office, these should be recorded locally and reported according to local SOPs.

\subsection{Reporting SAEs}

All SAEs, whether expected or otherwise from the day of consent and randomisation in to the trial until the defined end of the trial (as per section 10.3) should be reported. Whether observed directly or reported by the patient, these must be recorded on CRF 7 and faxed to BCTU on 0121 4159136 within 24 hours of the research staff becoming aware of the event. The Principal Investigator (or other nominated clinician) is required to assign seriousness, causality and expectedness to the SAE before reporting. All SAEs should be assessed for seriousness, causality and expectedness. The Chief Investigator will review the assessment of causality of all SAEs and 
provide guidance concerning causality assessment to site investigators. Any variations to those given by investigators will be fed back accordingly.

For each $\mathrm{SAE}$, the following information will be collected:

- full details in medical terms with a diagnosis, if possible

- its duration (start and end dates; times, if applicable)

- action taken

- outcome

- causality, in the opinion of the investigator*

- whether the event would be considered expected or unexpected* (using the principles described above)

*Assessment of causality and expectedness must be made by a clinician. If a clinician is unavailable, initial reports without causality and expectedness assessment should be submitted to BCTU by a healthcare professional within 24 hours, but must be followed up by medical assessment as soon as possible thereafter, ideally within the following 24 hours.

The local investigator and others responsible for patient care should institute any supplementary investigations of SAEs based on their clinical judgement of the likely causative factors and provide further follow-up information as soon as available.

SAEs still present beyond the defined end of trial must be followed up until the final outcome is determined.

BCTU will report all SAEs to the DMEC, the chief investigator and the trial sponsor, following a timetable agreed by the parties prior to study commencement. The DMEC will review these open, unblinded data for safety. BCTU will also report all SAEs to the main REC annually, and to the Trial Steering Committee, blinded to treatment allocation following a timetable agreed by the TSC prior to study commencement. Local Investigators are responsible for reporting SAEs to their host institution, according to local regulations, but they do not need to inform the main REC as this will be done by BCTU as detailed above.

\subsection{Safety reporting responsibilities}

Local Principal Investigator (or nominated individual in PI's absence):

- To record all SAEs that occur in the women taking part in the trial. This includes serious, expected or unexpected adverse events, unless defined as outcomes above.

- Medical judgement in assigning expectedness and causality to SAEs.

- To fax SAE forms to BCTU within 24 hours of becoming aware, and to provide further follow-up information as soon as available.

- To report SAEs to local committees if required, in line with local arrangements.

- To sign an Investigator's Agreement accepting these responsibilities. 


\section{Chief Investigator (or nominated individual in Cl's absence):}

- To assign causality and expected nature of SAEs where it has not been possible to obtain local assessment.

- To review all events assessed as SAEs in the opinion of the local investigator for causality and expectedness.

\section{Birmingham Clinical Trials Unit:}

- To prepare annual progress reports to the main REC and TSC.

- To prepare SAE safety reports for the DMEC following a timetable agreed by the DMEC prior to study commencement, or as requested by the DMEC.

- To report all fatal SAEs to the DMEC for continuous safety review.

\section{Trial Steering Committee (TSC):}

- To provide independent supervision of the scientific and ethical conduct of the trial on behalf of the Trial Sponsor and funding bodies.

- To review data, protocol deviations, outcome capture rates, adverse events (during treatment and up to the end of follow-up).

- To receive and consider any recommendations from the DMEC on protocol modifications.

\section{Data Monitoring \& Ethics Committee (DMEC):}

- To review (initially at approximately six-monthly intervals) overall safety and morbidity data to identify safety issues which may not be apparent on an individual case basis.

- To recommend to the TSC whether the trial should continue unchanged, continue with protocol modifications, or stop.

\section{Sponsor:}

- To report all related and unexpected SAEs to the REC according to SOPs and national timeframes.

- Take appropriate advice and recommendations from the TSC/DMEC in a timely manner to ensure safety of participants in the trial as well as the ethical and scientific integrity of the study.

\section{DATA MANAGEMENT}

\subsection{Clinical Data}

Data from the CRFs described in Section 8 should be entered into the secure online C-STICH database as soon as possible after collection by the research staff at the site. These personnel will be allocated personal usernames and passwords that will only allow access to entry forms for the trial participants who are being treated at their site. A copy of the forms can be sent to the C- 
STICH Trial Office for central input as described in Section 8. Data validation is built into the online database. Range, date and logic checks are performed at the point of data entry. Email reminders will be sent to the designated C-STICH staff members for missing data forms, missing data or data inconsistencies.

\subsection{Embedded microbiological sub-study}

\subsubsection{Specimen identification, processing and storage}

High vaginal swabs will be delivered to each participating hospital's local diagnostic microbiology laboratory where they will be processed in accordance with local standard operating procedures for routine processing of swabs or tissue. CRF 4 which collects data on the microbiology assessment includes a list of microorganisms that Microbiology Departments will be expected to identify in samples; this will ensure that there is consistency between centres in reporting the presence of bacteria that are not unequivocal pathogens.

Most laboratories would not routinely process suture materials. Therefore, laboratories should be asked to place the suture in 3-5 mL of sterile $0.9 \%$ saline. After sonication or vigorous vortexing, $0.1 \mathrm{~mL}$ volumes should be cultured on appropriate agar plates for culture for aerobic and anaerobic bacteria and fungi.

\subsubsection{Quality Assurance of Microbiological Assessments}

We will confirm that all contributing microbiology laboratories participate in an external quality assurance scheme and are accredited by CPA (UK) Ltd., UKAS, or another equivalent body.

\subsection{Definition of the End of Trial}

The study will be deemed complete when all neonates have reached the defined time points below. The end of neonatal follow up will be different for neonates born preterm compared with term.

For preterm neonates (born less than 37 weeks), the end of trial follow-up will be the estimated date of delivery (as confirmed by first trimester ultrasound) or discharge from hospital whichever occurred sooner.

For term neonates (born after 37 weeks), the end of trial follow up will be 28 days post-delivery or discharge from hospital whichever occurred sooner.

The end of trial for the mother will be 28 days post-delivery.

\section{ACCRUAL AND ANALYSIS}

\subsection{Sample size}

The sample size for C-STICH is informed by our meta-analysis (section 1.5) with some allowance made for the fact that this evidence is non-randomised. Here, the pregnancy loss rate was $7.1 \%$ with monofilament sutures compared to $19 \%$ with braided sutures, a reduction of $66 \%$ (RR: 0.34 , $95 \% \mathrm{Cl}: 0.18$ to 0.63 ; figure 1). A total sample of 326 women would be enough to detect a difference of this size (with $90 \%$ power and $p=0.05$ ), but we have inflated this to a total sample 
target of 900 (gaining full outcome data on 878) which will enable us to detect a more plausible relative reduction of $41 \%$ ( $19 \%$ with braided to $11.2 \%$ with monofilament) with $90 \%$ power $(p=0.05)$.

If the control rate of pregnancy loss in the braided group is lower than $19 \%$ then we still have reasonable power to detect this same relative difference of $41 \%$ provided the rate of pregnancy loss is at least $11 \%$ (Table 2 )

Table 2. Power calculation for C-STICH for various levels of pregnancy loss rate in the primary outcome

\begin{tabular}{|c|c|c|c|c|}
\hline \multicolumn{5}{|c|}{ For a relative reduction of 41\% (n=878) } \\
\hline $\begin{array}{c}\text { Rate in } \\
\text { monofilament } \\
\text { group }\end{array}$ & $\begin{array}{c}\text { Rate in braided } \\
\text { group }\end{array}$ & $\begin{array}{c}\text { Absolute risk } \\
\text { reduction }\end{array}$ & NNT & $\begin{array}{c}\text { Power } \\
(p=0.05)\end{array}$ \\
\hline 0.112 & 0.190 & 0.078 & 12.8 & $90 \%$ \\
\hline 0.100 & 0.170 & 0.070 & 14.3 & $86 \%$ \\
\hline 0.088 & 0.150 & 0.062 & 16.1 & $81 \%$ \\
\hline 0.077 & 0.130 & 0.053 & 18.9 & $73 \%$ \\
\hline 0.065 & 0.110 & 0.045 & 22.2 & $66 \%$ \\
\hline 0.053 & 0.090 & 0.037 & 27.0 & $57 \%$ \\
\hline
\end{tabular}

The control group rate of pregnancy loss will be monitored throughout the pilot and full study in conjunction with the DMEC to see how this may affect the sample size calculations. The DMEC will be given the remit of advising if our sample size would need to be altered based on this information.

\subsection{Projected accrual and attrition rates}

Hospital Episode Statistics data indicates that there are about 1950 procedures performed in England each year, from which you could extrapolate to estimate 2300 women per year in the UK as a whole undergo cerclage. Assuming $25 \%$ of these are ineligible, and assuming only $60 \%$ of the eligible women are approached and $50 \%$ of those consent, it is feasible to recruit 900 women in 30 months.

\subsection{Statistical Analysis}

The analysis will be by intention to treat. Every attempt will be made to gather data on all women randomised and their babies, irrespective of compliance with the treatment protocol. Point estimates, 95\% confidence intervals and $p$-values from two-sided tests will be calculated. A comprehensive Statistical Analysis Plan will be drawn up prior to any analysis and provided to the Independent Data Monitoring Committee (DMEC) for review.

\subsubsection{Primary analysis}

We will use a log-binomial regression model to calculate the relative risk and $95 \%$ confidence of the primary outcome (pregnancy loss defined as miscarriage or perinatal mortality). Minimisation variables (see section 5.1) will be included in the model as covariates. The statistical significance of the treatment group variable will be determined by an associated chi-squared test. 


\subsubsection{Secondary analysis}

Dichotomous secondary outcomes (e.g. infection, cervical tears, late neonatal death) will be analysed in the same fashion as the primary outcome. Time from conception to delivery and randomisation to delivery (censoring for pregnancy loss) will be analysed by log-rank test with a Cox Proportional Hazard (PH) model built if the assumptions of proportionality are met. Standard methods will be used to analyse other outcome (e.g. chi-squared test for mode of delivery). Appropriate summary statistics split by group will be presented for each outcome (e.g. proportions/percentages, mean/standard deviation or median/interquartile range).

\subsubsection{Sub-group analyses and missing data}

Subgroup analyses will be limited to the same variables which were used as minimisation variables (listed in section 5.1). Tests for statistical heterogeneity (e.g. by including treatment group by subgroup interaction parameter in the regression model) will be performed prior to any examination of effect estimate within subgroups. Sensitivity analyses will be performed on the primary outcome to investigate the impact of any missing data, e.g. assuming pregnancy loss for missing outcomes. Methods using multiple imputation (MI) will also be incorporated.

\subsubsection{Timing of assessments}

An interim report including the analysis of major endpoints will be provided in strict confidence to a Data Monitoring and Ethics Committee at intervals of at least 12 months, or to a timetable agreed by the DMEC prior to study commencement (see Section 12.5 for further details on trial data monitoring including the use of pragmatic stopping criteria). Final analysis will be performed once all live babies have reached the end of the follow-up period, as defined in Section 10.3.

\subsection{Confidentiality of personal data}

Personal and sensitive data will be collected directly from trial participants' hospital notes. Participants will be informed about the transfer of this information to the C-STICH Study Office at BCTU and asked for their consent. With the patient's consent, their initials, date of birth, National Health Service (NHS) or Community Health Index (CHI) number of both mother and baby, and Hospital number will be securely stored on the trial database. This will enable tracing of women who deliver in a different hospital.

Patients will be identified using only their unique trial number to verify identify on the data collection forms and in any correspondence between the C-STICH Study Office and the participating site.

Consent forms will be collected by the C-STICH Study Office and stored securely in the Trial Master File (TMF). These forms will be available to various regulatory bodies for inspection upon request.

Data collected will be entered onto a secure computer database, either directly by the local site via the internet using secure socket layer (SSL) encryption technology, or indirectly from paper forms by C-STICH study office staff. Access control will ensure that local trials staff will only be able to view information relating to participants at their site.

All personal information received in a paper format for the trial will be held securely in locked filing cabinets in a safe haven office and treated as strictly confidential according to BCTU policies. 
All staff involved in the C-STICH study, be they clinical, academic, or employees of BCTU, share the same duty of care to prevent unauthorised disclosure of personal information. No data that could be used to identify an individual will be published. Personal data recorded on all documents will be regarded as strictly confidential and will be handled and stored in accordance with the Data Protection Act 1998 and any amendments.

\subsection{Data Quality Assurance}

\subsubsection{Monitoring and Audit}

The study will be subject to onsite and remote monitoring to ensure compliance with GCP. A risk proportionate approach to the initiation, management and monitoring of the study will be adopted and outlined in the study-specific risk assessment. The sponsor will perform regular onsite monitoring for all sites according to BWH SOPs. The study specific risk assessment will be regularly reviewed in light of monitoring for all sites according to BWH SOPs. The study specific risk assessment will be regularly reviewed in light of monitoring findings to ensure risk proportionate levels of monitoring of data as per BCTU SOPs.

\subsubsection{Direct Access to Source Data}

Investigators and their host Trusts will be required to permit trial-related monitoring and audits to take place by the sponsor representative, providing direct access to source data and documents as requested. The trial site may also be subject to audit by the Research and Development Manager of their own Trust, or monitoring by the sponsor, and should do everything requested by the Chief Investigator in order to prepare and contribute to any inspection or audit or monitoring. Trial participants will be made aware of the possibility of external audit of data they provide in the participant information sheet.

\subsubsection{Central monitoring throughout the trial}

The study will also adopt a centralised approach to monitoring data quality and compliance. A computer database will be constructed specifically for the trial data and will include range and logic checks to prevent erroneous data entry. Independent checking of data entry will be periodically undertaken on small sub-samples. The trial statistician will regularly check the balance of allocations by the stratification variables.

\subsection{Definition of a serious breech}

A serious breach is that which is likely to effect to a significant degree:

1. The safety or physical or mental integrity of the participants of the trial; or

2. The scientific value of the trial.

If a potential serious breach is identified by the Chief investigator, Principal Investigator or BCTU, the C-STICH Trial Office must be notified within 24 hours. It is the responsibility of the Chief Investigator to determine whether the incident constitutes a serious breach and if so, to assess the impact of the breach on the scientific value of the trial. BCTU will report serious breaches to the sponsor and to the Research Ethics Committee as necessary. 


\subsection{Trial Steering Committee}

The TSC provides independent supervision for the trial, providing advice to the Chief and CoInvestigators and the sponsor on all aspects of the trial and affording protection for patients by ensuring the trial is conducted according to the guidelines for Good Clinical Practice.

If the Chief and Co-Investigators are unable to resolve any concern satisfactorily, Principal Investigators, and all others associated with the study may write, through the Trial Office, to the chairman of the TSC drawing attention to any concerns they may have about the possibility of particular side-effects, of particular categories of patient requiring special study, or any other matters thought relevant.

\subsection{Data Monitoring and Ethics Committee:} determining when clear answers have emerged

If one treatment really is substantially better or worse than any other with respect to the primary outcome, then this may become apparent before the target recruitment has been reached. Alternatively, new evidence might emerge from other sources that any one treatment is definitely more, or less, effective than any other. To protect against this, during the main period of recruitment to the study, interim analyses of the primary outcome and adverse events will be supplied, in strict confidence, to an independent Data Monitoring and Ethics Committee (DMEC) along with updates on results of other related studies, and any other analyses that the DMEC may request. The DMEC will advise the chair of the TSC if, in their view, any of the randomised comparisons in the trial have provided both (a) "proof beyond reasonable doubt" that for all, or for some, types of patient one particular treatment is definitely indicated or definitely contraindicated in terms of a net difference in the major endpoints, and (b) evidence that might reasonably be expected to influence the patient management of many clinicians who are already aware of the other main trial results. The TSC can then decide whether to close or modify any part of the trial. Unless this happens, however, the TMG, TSC, the investigators and all of the central administrative staff (except the statisticians who supply the confidential analyses) will remain unaware of the interim results.

The BCTU Trial office will forward open DMEC meeting minutes to the Sponsor and Funding Body.

Appropriate criteria of proof beyond reasonable doubt cannot be specified precisely, but a difference of at least $p<0.001$ (similar to a Haybittle-Peto stopping boundary) in an interim analysis of a major endpoint may be needed to justify halting, or modifying, the study prematurely. If this criterion were to be adopted, it would have the practical advantage that the exact number of interim analyses would be of little importance, so no fixed schedule is proposed.

\subsection{Long-term storage of data}

Archiving will be authorised by BCTU on behalf of the Sponsor following submission of the end of trial report. Destruction of essential documents will require authorisation from the Sponsor.

Principal Investigators are responsible for the secure archiving of essential trial documents for their site, according to the local policy at that site. All essential documents will be archived for a minimum of 5 years after completion of trial. Destruction of essential documents will require authorisation from the Sponsor. 
Trial data will be stored under controlled conditions for at least 3 years after closure. This will allow adequate time for review and reappraisal, and in particular with the C-STICH trial, form the basis for further follow-up research. Any queries or concerns about the data, conduct or conclusions of the trial can also be resolved in this time. Long-term offsite data archiving facilities will be considered for storage after this time. BCTU has standard processes for both hard copy and computer database legacy archiving, including anonymisation of trial data.

\section{ORGANISATION AND RESPONSIBILITIES}

To ensure the smooth running of the trial and to minimise the overall procedural workload, it is proposed that each participating centre should designate individuals who would be chiefly responsible for local co-ordination of clinical and administrative aspects of the trial. This includes delegation to appropriately qualified site staff to carry out informed consent procedures.

All investigators are responsible for ensuring that any research they undertake follows the agreed protocol, for helping care professionals to ensure that participants receive appropriate care while involved in research, for protecting the integrity and confidentiality of clinical and other records and data generated by the research, and for reporting any failures in these respects, adverse reactions and other events or suspected misconduct through the appropriate systems.

\subsection{Centre eligibility}

Centres will be eligible to participate in C-STICH if they routinely perform cervical cerclage with or without bladder dissection, and are prepared to randomise between monofilament and braided cerclage sutures.

\subsection{Local Co-ordinator at each centre}

Each Centre should nominate an obstetrician or gynaecologist or other appropriate clinical staff member to act as the local Principal Investigator and bear responsibility for the conduct of research at their centre. Close collaboration between all clinical teams is particularly important in C-STICH in order that patients for whom cervical cerclage is an appropriate treatment option can be identified sufficiently early for inclusion in the trial.

The local Principal Investigator is responsible for the overall conduct of the study at the site and to ensure compliance with the protocol and any amendments. In accordance with the principles of International Committee on Harmonisation Good Clinical Practice Guidelines (ICH GCP) the following areas listed in this section are also the responsibility of each Investigator. Duties may be delegated to an appropriate member of study site staff. Delegated tasks must be documented on a Delegation Log and signed by all those named on the list prior to undertaking applicable studyrelated procedures:

- to ensure that all medical and nursing staff involved in the care of women deemed at risk of cervical insufficiency are well informed about the study and trained in trial procedures

- to ensure written informed consent is obtained before randomisation

- to designate or recruit a C-STICH research staff member

- to be responsible for the quality of data recorded in the data collection forms at their site 
- to maintain their site's Investigator Site File

- to sign the Investigator's Declaration in the Clinical Study Site Agreement

- to ensure all study staff hold evidence of appropriate GCP training

- to ensure confidentiality of all trial data collected

- to report to the C-STICH Trial Office all SAEs in a timely manner

- to report any protocol violations and suspected serious breaches to the C-STICH trial office

- to comply with the C-STICH Protocol

\subsection{Designated C-STICH staff member/s at each centre}

Each participating centre should also designate one staff member as local Nursing / Midwifery / Research Coordinator. This person would be responsible for ensuring that all eligible patients are considered for the study, that patients are provided with study information sheets, and have an opportunity to discuss the study if required. The staff member may be responsible for collecting the baseline patient data and for administering the follow-up evaluations. This person would be sent updates and newsletters, and would be invited to training and progress meetings.

\subsection{The C-STICH Trials Office at BCTU}

The C-STICH Trial Office at BCTU is responsible for providing all trial documentation, including the trial folders containing printed documents and the update slides. These will be supplied to each collaborating centre after all relevant approvals have been obtained. Additional supplies of any printed material can be obtained on request. The Trial Office also provides the central randomisation service and is responsible for collection and checking of data (including reports of serious adverse events thought to be due to trial treatment), for reporting of serious and unexpected adverse events to the sponsor and/ or regulatory authorities and for analyses. The Trial Office will help resolve any local problems that may be encountered in trial participation.

\subsection{Research Governance}

The conduct of the trial will be according to the principles of the International Committee on Harmonisation, Good Clinical Practice Guidelines (ICH GCP).

All centres will be required to sign an Investigator's Agreement, detailing their commitment to accrual, compliance, Good Clinical Practice, confidentiality and publication. Deviations from the agreement will be monitored and the TSC will decide whether any action needs to be taken, e.g. withdrawal of funding, suspension of centre.

The Trial Office will ensure researchers not employed by an NHS organisation hold an NHS honorary contract for that organisation. 


\subsection{Regulatory and Ethical Approval}

\subsubsection{Ethical and Trust Management Approval or Confirmation of Capacity and Capability}

The Trial has a favourable ethical opinion from Cambridgeshire and Hertfordshire Multi-centre Research Ethics Committee (REC), confirming that the trial design respects the rights, safety and wellbeing of the participants. The Comprehensive Research Network will conduct governance checks and assess the facilities and resources needed to run the trial, in order to give host site permission. For sites in Scotland and Wales, the Trial Office is able to help the local Principal Investigator in the process of the site specific assessment by completing much of Site Specific Information section of the standard IRAS form as possible. For sites in England, the trial office is able to help with Trust confirmation of capacity and capability by helping to complete the Statement of Activities and Schedule of Events. The local Principal Investigator will be responsible for liaison with the Trust management with respect to locality issues and obtaining the necessary signatures at their Trust.

Once the following has taken place for each Trust, Health Board, or NHS Board, the Trial Office will send a folder containing all trial materials to the local Principal Investigator along with a letter of activation, and potential trial participants can then start to be approached:

Sites in Scotland have received NHS Board permission for the study

Sites in Wales have received Health Board approval for the study

Sites in England have received Trust confirmation of Capacity and Capability

The contract agreement for the Trust/Health Board/NHS Board is fully executed

The site initiation visit has taken place

A delegation log has been completed by the PI and sent to the trial office

A CV and GCP Certificate for the PI has been sent to the trial office

Within 90 days after the end of the study, the Chief Investigator will on behalf of the Sponsor, ensure that the REC is notified that the study has finished. If the study is terminated prematurely, those reports will be made within 15 days after the end of the study.

The Chief Investigator will supply the Sponsor with a summary report of the clinical study, which will then be submitted to the REC within one year after the end of the study.

\subsection{Funding and Cost implications}

The research costs of the trial are funded by a grant from the NIHR Health Technology Assessment Programme awarded to the BWCNFT.

The trial has been designed to minimise extra 'service support' costs for participating hospitals, with no extra visits to hospital and no extra tests. Additional costs service support costs associated with the trial, e.g. identifying potential participants, gaining consent, are estimated in the Contract agreement. These costs should be met by accessing the Trust's Support for Science budget via the Comprehensive Research Network. 


\subsection{Indemnity}

This is a clinician-initiated study. The Sponsor (the BWCNFT) holds the relevant insurance for Clinical Trials (negligent harm). Participants may be able to claim compensation, if they can prove that the BWCNFT has been negligent. However, as this clinical trial is being carried out in a hospital setting, NHS Trust and Non-Trust Hospitals have a duty of care to the patients being treated. Compensation is only available via NHS indemnity in the event of clinical negligence being proven. Participants who sustain injury and wish to make a claim for compensation should do so in writing in the first instance to the $\mathrm{Cl}$, who will pass the claim to the Sponsor's Insurers, via the Sponsor's office. There are no specific arrangements for compensation made in respect of any SAE occurring though participation in the trial, whether from the side effects listed, or others yet unforeseen.

Hospitals selected to participate in this trial shall provide clinical negligence insurance cover for harm caused by their employees and a copy of the relevant insurance policy or summary should be provided to BWCNFT, upon request.

\section{REPORTING, PUBLICATIONS AND NOTIFICATION OF RESULTS}

\subsection{AUTHORSHIP POLICY}

Ownership of the data arising from this study resides with the grant holders. On completion of the study, the study data will be analysed and tabulated, and a final study report prepared for the NIHR. A writing committee may be established to prepare the report and any subsequent papers.

The main report of the trial will be published in the name of the C-STICH Collaborative Group, acknowledging the writing group as authors. Subsequent publications should also be published in the C-STICH Collaborative Group name, but those academics who contribute to specific aspects may be listed as authors.

\subsection{PUBLICATION}

A meeting will be held after the end of the study to allow discussion of the main results among the collaborators prior to publication. The success of the study depends entirely on the wholehearted collaboration of a large number of clinicians, Nurses / Midwifes and others. For this reason, chief credit for the main results will be given not to the committees or central organisers but to all those who have collaborated in the study. Centres will be permitted to publish data obtained from participants in the C-STICH Trial that use Trial outcome measures but do not relate to the trial randomised evaluation and hypothesis.

\subsection{Ancillary studies}

It is requested that any proposals for formal additional studies of the effects of the trial treatments on some patients (e.g. special investigations in selected hospitals) be referred to the Trial Management Group for consideration. In general, it would be preferable for the trial to be kept as simple as possible, and add-on studies will need to be fully justified. 


\section{REFERENCES}

1) Office for National Statistics. Preterm births, preterm births data, press release based on 2005 data. Available at: www.ons.gov.uk/ons/publications/re-referencetables.html?edition=tcm\%3A77-50818 (2007, accessed 26 November 2012).

2) Preterm birth: what can be done? Lancet. 2008; 371(9606): 2.

3) Lewitt EM, Baker LS, Corman H, Shiono PH. The direct cost of low birthweight. The future of children. Los Altos, CA: David and Lucile Packard Foundation, 1995:35-56.

4) Shirodkar VN. A new method of operative treatment for habitual abortions in 183 the second trimester of pregnancy. Antiseptic 1955;52:299.

5) McDonald IA. Incompetent cervix as a cause of recurrent abortion. J Obstet Gynaecol Br Common. 1963; 70(105).

6) McDonald IA. Cervical cerclage. Clinics in obstetrics and gynaecology. 1980; 7(3): 461-79.

7) F. Israfil-Bayli, P. Toozs-Hobson, C. Lees, M. Slack, J. Daniels, A. Vince, and K. M. K. Ismail. Cervical cerclage and type of suture material: a survey of UK consultants' practice. Journal of Maternal-Fetal and Neonatal Medicine. 2014,27 (15): 1584-1588.

8) Royal College of Obstetricians and Gynaecologists (RCOG). Cervical cerclage. London (UK). 2011 May, 21 p. (Green-top guideline; no 60).

9) Alfirevic Z, Stampalija T, Roberts D, Jorgensen AL. Cervical stitch (cerclage) for preventing preterm birth in singleton pregnancy. Cochrane Database Syst Rev. 2012; 4:CD008991.

10) Van Winkle W, Jr., Hastings JC, Barker E, Hines D, Nichols W. Effect of suture materials on healing skin wounds. Surgery, gynecology \& obstetrics. 1975; 140(1): 7-12.

11) Amid PK, Shulman AG, Lichtenstein IL, Hakakha M. Biomaterials for abdominal wall hernia surgery and principles of their applications. Langenbecks Archiv fur Chirurgie.1994; 379(3):168-71.

12) Slack M, Sandhu JS, Staskin DR, Grant RC. In vivo comparison of suburethral sling materials. International Urogynecology journal and pelvic floor dysfunction. $2006 \mathrm{Feb} ; 17(2): 106-10$.

13) Visco AG, Weidner AC, Barber MD, Myers ER, Cundiff GW, Bump RC, et al. Vaginal mesh erosion after abdominal sacral colpopexy. American journal of obstetrics and gynecology. 2001 Feb;184(3):297-302.

14) Blomstedt B, Osterberg B. Fluid absorption and capillarity of suture materials. Acta chirurgica Scandinavica. 1977;143(2):67-70.

15) Blomstedt B, Osterberg B, Bergstrand A. Suture material and bacterial transport. An experimental study. Acta chirurgica Scandinavica. 1977;143(2):71-3.

16) Wohlrab KJ, Erekson EA, Myers DL. Postoperative erosions of the Mersilene ${ }^{\circledR}$ suburethral sling mesh for anti-incontinence surgery. International urogynecology journal and pelvic floor dysfunction. 2009 Apr;20(4):417-20. PubMed PMID: 19082911.

17) Royal College of Obstetricians and Gynaecologists. Scientific Impact Paper Number 19. The Use of Mesh in Gynaecological Surgery. RCOG. April 2010.

18) Laparoscopic cerclage for prevention of recurrent pregnancy loss due to cervical incompetence. NICE interventional procedures guidance [IPG228] Published date: August 2007. 


\section{Example Patient Pathway}

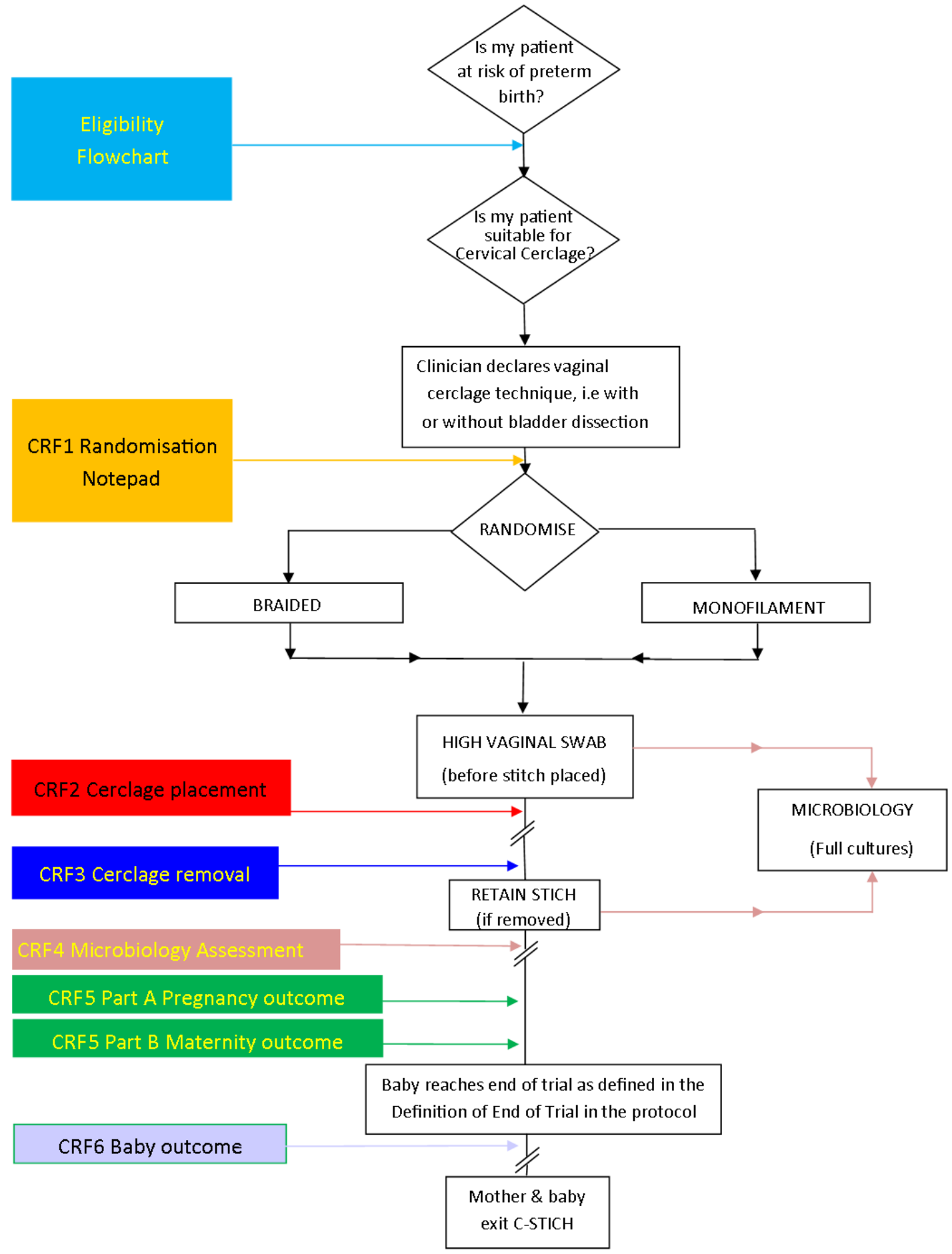


Cerclage Suture Type for an Insufficient Cervix and its effect on Health outcomes (C-STICH)

\section{Is my patient suitable for C-STICH?}

The flowchart below will quickly let you know if your patient is eligible for the C-STICH trial.

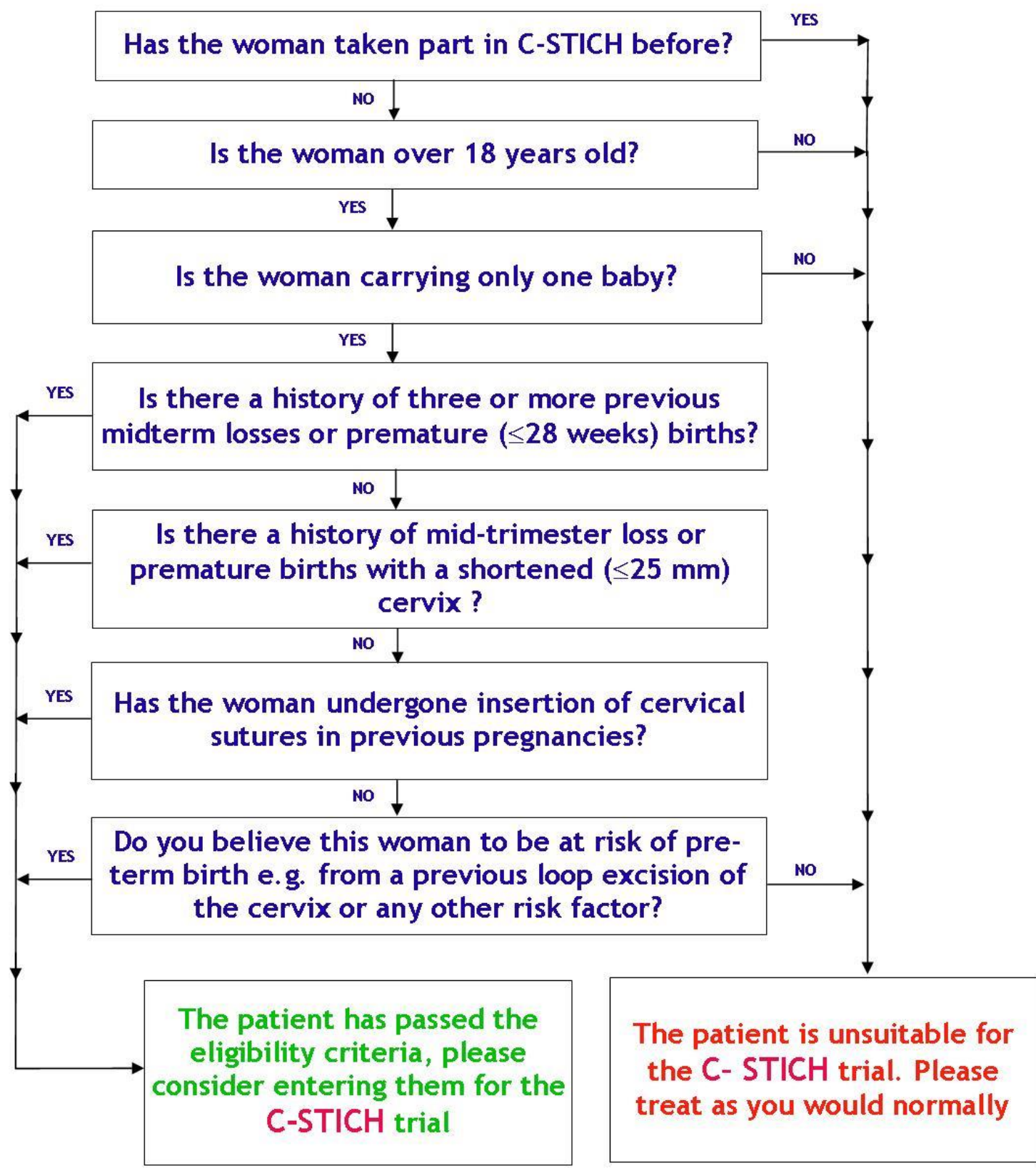

